\title{
Weak decays of doubly heavy baryons: The FCNC processes
}

\author{
Zhi-Peng Xing and Zhen-Xing Zhao* \\ INPAC, Shanghai Key Laboratory for Particle Physics and Cosmology, MOE Key Laboratory for Particle \\ Physics, Astrophysics and Cosmology, School of Physics and Astronomy, Shanghai Jiao-Tong University, \\ Shanghai 200240, People's Republic of China
}

(Received 15 July 2018; published 5 September 2018)

\begin{abstract}
The discovery of doubly heavy baryon provides us with a new platform for precisely testing the standard model and searching for new physics. As a continuation of our previous works, we investigate the flavorchanging neutral current processes of doubly heavy baryons. The light-front approach is adopted to extract the form factors, in which the two spectator quarks are viewed as a diquark. Results for form factors are then used to predict some phenomenological observables, such as the decay width and the forwardbackward asymmetry. We find that most of the branching ratios for $b \rightarrow s$ processes are $10^{-8}-10^{-7}$ and those for $b \rightarrow d$ processes are $10^{-9}-10^{-8}$. The flavor SU(3) symmetry and symmetry breaking effects are explored. Parametric uncertainties are also investigated.
\end{abstract}

DOI: 10.1103/PhysRevD.98.056002

\section{INTRODUCTION}

Just one year ago, LHCb collaboration announced the discovery of a doubly charmed baryon $\Xi_{c c}^{++}$with the mass [1]

$$
m_{\Xi_{c c}^{++}}=(3621.40 \pm 0.72 \pm 0.27 \pm 0.14) \mathrm{MeV} .
$$

Since then, great theoretical interests have been devoted to the study of doubly heavy baryons, some of them can be found in Refs. [2-22]. Recently, some more new results were reported on $\Xi_{c c}^{++}$by LHCb collaboration, including the first measurement of the lifetime [23] and the first observation of the new decay mode $\Xi_{c c}^{++} \rightarrow \Xi_{c}^{+} \pi^{+}$[24]. After discovering $\Xi_{c c}^{++}$in the decay mode of $\Xi_{c c}^{++} \rightarrow$ $\Lambda_{c}^{+} K^{-} \pi^{+} \pi^{+}$, LHCb collaboration is also continuing to search for the $\Xi_{c c}^{+}$and $\Xi_{b c}$ baryons [25]. Comprehensive theoretical studies on weak decays are highly demanded and our previous and forthcoming works aim to fill this gap. In our previous works [4,5], we have presented the calculations of $1 / 2$ to $1 / 2$ and $1 / 2$ to $3 / 2$ weak decays. As a continuation, we investigate the flavor-changing neutral current (FCNC) processes in this work.

FCNC processes are considered to be an ideal place to the precise test of the standard model (SM) and the search for new physics (NP), while the discovery of the doubly

\footnotetext{
*star_0027@sjtu.edu.cn
}

Published by the American Physical Society under the terms of the Creative Commons Attribution 4.0 International license. Further distribution of this work must maintain attribution to the author(s) and the published article's title, journal citation, and DOI. Funded by SCOAP. heavy baryon provides us a new platform. $b \rightarrow d / s$ process in SM is induced by the loop effect, thus its decay width is small. NP effects manifest themselves in two different ways. One is to enhance the Wilson coefficients, and the other is to introduce new effective operators which are absent in the SM. The typical value of the branching ratio for FCNC processes is $\sim 10^{-6}$ for mesonic sector. However, the small branching ratio can be compensated by the high luminosity at the $B$ factories. Also, with the accumulation of data, we are in an increasingly better position to study these semileptonic process. Baryonic rare decay modes, which are also induced by $b \rightarrow d / s l^{+} l^{-}$at the quark level, are also important as its mesonic counterparts. Serious attention is deserved, both theoretically and experimentally.

A doubly heavy baryon is composed of two heavy quarks and one light quark. Light flavor SU(3) symmetry arranges them into the presentation 3 . For $1 / 2^{+}$doubly heavy baryons, we have $\Xi_{c c}^{++,+}$and $\Omega_{c c}^{+}$in the $c c$ sector, $\Xi_{b b}^{0,-}$ and $\Omega_{b b}^{-}$in the $b b$ sector, while there are two sets of baryons in the $b c$ sector depending on the symmetric property under the interchange of $b$ and $c$ quarks. For the symmetric case, the set is denoted by $\Xi_{b c}^{+, 0}$ and $\Omega_{b c}^{0}$, while for the asymmetric case, the set is denoted by $\Xi_{b c}^{\prime+, 0}$ and $\Omega_{b c}^{\prime 0}{ }^{1}{ }^{1}$ In reality these two sets probably mix with each other, which will not be considered in this work.

To be explicit, we will concentrate on the following FCNC decay modes of doubly heavy baryons. For $b \rightarrow s$ process,

${ }^{1}$ The convention here for $b c$ sector is opposite to that in Ref. [26]. 
(i) $b b$ sector

$$
\begin{aligned}
& \Xi_{b b}^{0}(b b u) \rightarrow \Xi_{b}^{0}(s b u) / \Xi_{b}^{\prime 0}(s b u), \\
& \Xi_{b b}^{-}(b b d) \rightarrow \Xi_{b}^{-}(s b d) / \Xi_{b}^{\prime-}(s b d), \\
& \Omega_{b b}^{-}(b b s) \rightarrow \Omega_{b}^{-}(s b s),
\end{aligned}
$$

(ii) $b c$ sector

$$
\begin{aligned}
& \Xi_{b c}^{+}(b c u) / \Xi_{b c}^{\prime+}(b c u) \rightarrow \Xi_{c}^{+}(s c u) / \Xi_{c}^{\prime+}(s c u), \\
& \Xi_{b c}^{0}(b c d) / \Xi_{b c}^{\prime 0}(b c d) \rightarrow \Xi_{c}^{0}(s c d) / \Xi_{c}^{\prime 0}(s c d), \\
& \Omega_{b c}^{0}(b c s) / \Omega_{b c}^{\prime 0}(b c s) \rightarrow \Omega_{c}^{0}(s c s) .
\end{aligned}
$$

For $b \rightarrow d$ process,

(i) $b b$ sector

$$
\begin{aligned}
& \Xi_{b b}^{0}(b b u) \rightarrow \Lambda_{b}^{0}(d b u) / \Sigma_{b}^{0}(d b u), \\
& \Xi_{b b}^{-}(b b d) \rightarrow \Sigma_{b}^{-}(d b d), \\
& \Omega_{b b}^{-}(b b s) \rightarrow \Xi_{b}^{-}(d b s) / \Xi_{b}^{\prime-}(d b s),
\end{aligned}
$$

(ii) $b c$ sector

$$
\begin{aligned}
& \Xi_{b c}^{+}(b c u) / \Xi_{b c}^{+}(b c u) \rightarrow \Lambda_{c}^{+}(d c u) / \Sigma_{c}^{+}(d c u), \\
& \Xi_{b c}^{0}(b c d) / \Xi_{b c}^{\prime 0}(b c d) \rightarrow \Sigma_{c}^{0}(d c d), \\
& \Omega_{b c}^{0}(b c s) / \Omega_{b c}^{\prime 0}(b c s) \rightarrow \Xi_{c}^{0}(d c s) / \Xi_{c}^{\prime 0}(d c s) .
\end{aligned}
$$

In the above, the quark components of the baryons have been explicitly presented in the brackets, and the quarks that participate in weak decay are put in the first place. Taking the $b \rightarrow s$ process in $b c$ sector as an example, the final baryons $\Xi_{c}^{+, 0}$ belong to the presentation of $\overline{\mathbf{3}}$, while $\Xi_{c}^{\prime+, 10}$ and $\Omega_{c}^{0}$ belong to the presentation of $\mathbf{6}$, as can be seen from Fig. 1.

Light front approach will be adopted to deal with the dynamics in the decay. This method has been widely used to study the mesonic decays [27-44]. Its application to baryonic sector can be found in Refs. [45-49]. As in our previous works, diquark picture is once again adopted, i.e., the two spectator quarks are viewed as a whole system, as can be seen from Fig. 2. The two spectator quarks form a scalar diquark or an axial-vector diquark. Generally speaking, both types of diquarks contribute to the decay process

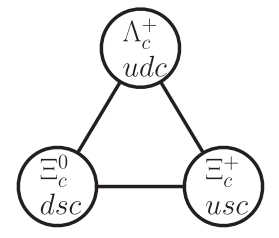

(a)

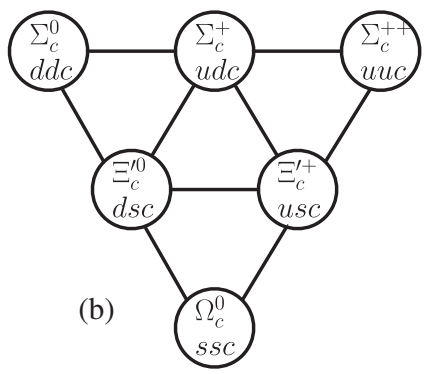

FIG. 1. Spin-1/2 anti-triplets (panel a) and sextets (panel b) of charmed baryons. It is similar for the bottomed baryons.

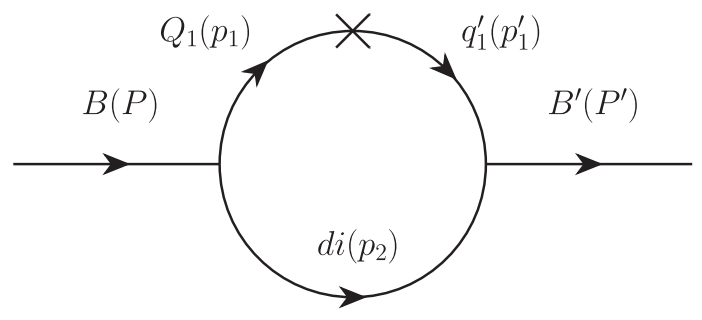

FIG. 2. Feynman diagram for baryon-baryon transition in the diquark picture. $P^{(\prime)}$ is the momentum of the parent (daughter) baryon, $p_{1}^{(\prime)}$ is the initial (final) quark momentum, $p_{2}$ is the diquark momentum, and the cross mark denotes the weak interaction.

and their contribution weights can be determined by the wave functions of the baryons in the initial and final states.

SU(3) analyses for FCNC processes will also be conducted. A quantitative predictions of $\mathrm{SU}(3)$ symmetry breaking effects will be performed within the light-front approach.

The rest of the paper is arranged as follows. In Sec. II, we will present the effective Hamiltonian responsible for the $b \rightarrow d / s l^{+} l^{-}$process. Then the framework of light-front approach under the diquark picture will be briefly introduced, then flavor-spin wave functions will also be discussed. Some phenomenological observables are collected in the last subsection of Sec. II. Numerical results are shown in Sec. III, including the results for form factors, decay widths, forward-backward asymmetry, the SU(3) symmetry breaking and the error estimates. A brief summary is given in the last section.

\section{THEORETICAL FRAMEWORK}

\section{A. The effective Hamiltonian}

The effective Hamiltonian for $b \rightarrow s l^{+} l^{-}$is given as

$$
\mathcal{H}_{\mathrm{eff}}\left(b \rightarrow s l^{+} l^{-}\right)=-\frac{G_{F}}{\sqrt{2}} V_{t b} V_{t s}^{*} \sum_{i=1}^{10} C_{i}(\mu) O_{i}(\mu) .
$$

Here the explicit forms of the four-quark and the penguin operators $O_{i}$ can be found in Ref. [50] and $C_{i}$ are their corresponding Wilson coefficients, which are presented in Table I in the leading logarithm approximation [50]. The transition amplitude for $\mathcal{B} \rightarrow \mathcal{B}^{\prime} l^{+} l^{-}$turns out to be

$$
\begin{aligned}
\mathcal{M}\left(\mathcal{B} \rightarrow \mathcal{B}^{\prime} l^{+} l^{-}\right)= & -\frac{G_{F}}{\sqrt{2}} V_{t b} V_{t s}^{*} \frac{\alpha_{\mathrm{em}}}{2 \pi} \\
& \times\left\{\left(C_{9}^{\mathrm{eff}}\left(q^{2}\right)\left\langle\mathcal{B}^{\prime}\left|\bar{s} \gamma_{\mu}\left(1-\gamma_{5}\right) b\right| \mathcal{B}\right\rangle\right.\right. \\
& \left.-2 m_{b} C_{7}^{\mathrm{eff}}\left\langle\mathcal{B}^{\prime}\left|\bar{s} i \sigma_{\mu \nu} \frac{q^{\nu}}{q^{2}}\left(1+\gamma_{5}\right) b\right| \mathcal{B}\right\rangle\right) \bar{l} \gamma^{\mu} l \\
& \left.+C_{10}\left\langle\mathcal{B}^{\prime}\left|\bar{s} \gamma_{\mu}\left(1-\gamma_{5}\right) b\right| \mathcal{B}\right\rangle \bar{l} \gamma^{\mu} \gamma_{5} l\right\}
\end{aligned}
$$


TABLE I. Wilson coefficients $C_{i}\left(m_{b}\right)$ calculated in the leading logarithmic approximation, with $m_{W}=80.4 \mathrm{GeV}$ and $\mu=m_{b, \text { pole }}[50]$.

\begin{tabular}{lcccccccc}
\hline \hline$C_{1}$ & $C_{2}$ & $C_{3}$ & $C_{4}$ & $C_{5}$ & $C_{6}$ & $C_{7}^{\text {eff }}$ & $C_{9}$ & $C_{10}$ \\
\hline 1.107 & -0.248 & -0.011 & -0.026 & -0.007 & -0.031 & -0.313 & 4.344 & -4.669 \\
\hline \hline
\end{tabular}

Note that the sign before $C_{7}^{\text {eff }}$ is different in literatures. Our result coincides with those in Refs. [51,52], but is different from that in Ref. [53]. In Eq. (3), $C_{7}^{\text {eff }}$ and $C_{9}^{\text {eff }}$ are defined by [54]

$$
\begin{aligned}
C_{7}^{\mathrm{eff}}= & C_{7}-C_{5} / 3-C_{6}, \\
C_{9}^{\mathrm{eff}}\left(q^{2}\right)= & C_{9}(\mu)+h\left(\hat{m}_{c}, \hat{s}\right) C_{0} \\
& -\frac{1}{2} h(1, \hat{s})\left(4 C_{3}+4 C_{4}+3 C_{5}+C_{6}\right) \\
& -\frac{1}{2} h(0, \hat{s})\left(C_{3}+3 C_{4}\right) \\
& +\frac{2}{9}\left(3 C_{3}+C_{4}+3 C_{5}+C_{6}\right),
\end{aligned}
$$

with $\hat{s}=q^{2} / m_{b}^{2}, C_{0}=C_{1}+3 C_{2}+3 C_{3}+C_{4}+3 C_{5}+C_{6}$, and $\hat{m}_{c}=m_{c} / m_{b}$. The auxiliary functions $h$ are given by

$$
\begin{aligned}
h(z, \hat{s})= & -\frac{8}{9} \ln \frac{m_{b}}{\mu}-\frac{8}{9} \ln z+\frac{8}{27}+\frac{4}{9} x-\frac{2}{9}(2+x)|1-x|^{1 / 2} \\
& \times \begin{cases}\left(\ln \left|\frac{\sqrt{1-x}+1}{\sqrt{1-x}-1}\right|-i \pi\right), & x \equiv \frac{4 z^{2}}{\hat{s}}<1 \\
2 \arctan \frac{1}{\sqrt{x-1}}, & x \equiv \frac{4 z^{2}}{\hat{s}}>1\end{cases} \\
h(0, \hat{s})= & -\frac{8}{9} \ln \frac{m_{b}}{\mu}-\frac{4}{9} \ln \hat{s}+\frac{8}{27}+\frac{4}{9} i \pi
\end{aligned}
$$

The effective Hamiltonian and transition amplitude for $b \rightarrow d$ process can be written down in a similar way.

\section{B. Light-front approach}

Light-front approach for $1 / 2 \rightarrow 1 / 2$ FCNC transition will be briefly introduced in this subsection, including the definitions of the states for spin-1/2 baryons, and the extraction of form factors. More details can be found in Refs. [45,49].

In the light-front approach, the wave function of $1 / 2^{+}$baryon with a scalar or an axial-vector diquark is expressed as

$$
\begin{aligned}
& \left|\mathcal{B}\left(P, S, S_{z}\right)\right\rangle \\
& =\int\left\{d^{3} p_{1}\right\}\left\{d^{3} p_{2}\right\} 2(2 \pi)^{3} \delta^{3}\left(\tilde{P}-\tilde{p}_{1}-\tilde{p}_{2}\right) \\
& \quad \times \sum_{\lambda_{1}, \lambda_{2}} \Psi S S_{z}\left(\tilde{p}_{1}, \tilde{p}_{2}, \lambda_{1}, \lambda_{2}\right)\left|q_{1}\left(p_{1}, \lambda_{1}\right)(d i)\left(p_{2}, \lambda_{2}\right)\right\rangle,
\end{aligned}
$$

where $q_{1}$ stands for $b / s$ quark in the initial/final state, and the diquark is denoted by $(d i)$, which is composed of one $b$ quark and one light quark. The momentum-space wave function $\Psi S S_{z}$ is given as

$$
\begin{aligned}
\Psi^{S S_{z}}\left(\tilde{p}_{1}, \tilde{p}_{2}, \lambda_{1}, \lambda_{2}\right)= & \frac{A}{\sqrt{2\left(p_{1} \cdot \bar{P}+m_{1} M_{0}\right)}} \\
& \times \bar{u}\left(p_{1}, \lambda_{1}\right) \Gamma u\left(\bar{P}, S_{z}\right) \phi\left(x, k_{\perp}\right),
\end{aligned}
$$

with $A=1$ and $\Gamma=1$ for the case of a scalar diquark involved, and $A=\sqrt{\frac{3\left(m_{1} M_{0}+p_{1} \cdot \bar{P}\right)}{3 m_{1} M_{0}+p_{1} \cdot \bar{P}+2\left(p_{1} \cdot p_{2}\right)\left(p_{2} \cdot \bar{P}\right) / m_{2}^{2}}}$ and $\Gamma=$ $-\frac{1}{\sqrt{3}} \gamma_{5} \phi^{*}\left(p_{2}, \lambda_{2}\right)$ for the case of an axial-vector diquark involved. A Gaussian-type function is usually adopted for $\phi$ :

$$
\phi=4\left(\frac{\pi}{\beta^{2}}\right)^{3 / 4} \sqrt{\frac{e_{1} e_{2}}{x_{1} x_{2} M_{0}}} \exp \left(\frac{-\vec{k}^{2}}{2 \beta^{2}}\right) .
$$

Taking the $V-A$ current of $b \rightarrow s$ process as an example, the transition matrix element can be derived as

$$
\begin{aligned}
& \left\langle\mathcal{B}^{\prime}\left(P^{\prime}, S_{z}^{\prime}\right)\left|\bar{s} \gamma_{\mu}\left(1-\gamma_{5}\right) b\right| \mathcal{B}\left(P, S_{z}\right)\right\rangle \\
& =\int\left\{d^{3} p_{2}\right\} \frac{\varphi^{\prime}\left(x^{\prime}, k_{\perp}^{\prime}\right) \varphi\left(x, k_{\perp}\right)}{2 \sqrt{p_{1}^{+} p_{1}^{\prime+}\left(p_{1} \cdot \bar{P}+m_{1} M_{0}\right)\left(p_{1}^{\prime} \cdot \bar{P}^{\prime}+m_{1}^{\prime} M_{0}^{\prime}\right)}} \\
& \times \sum_{\lambda_{2}} \bar{u}\left(\bar{P}^{\prime}, S_{z}^{\prime}\right) \bar{\Gamma}^{\prime}\left(\not p_{1}^{\prime}+m_{1}^{\prime}\right) \gamma_{\mu}\left(1-\gamma_{5}\right)\left(\not p_{1}+m_{1}\right) \Gamma u\left(\bar{P}, S_{z}\right),
\end{aligned}
$$

where

$$
m_{1}=m_{b}, \quad m_{1}^{\prime}=m_{s}, \quad m_{2}=m_{(d i)},
$$

and $\varphi^{(\prime)}=A^{(\prime)} \phi^{(\prime)}, p_{1}\left(p_{1}^{\prime}\right)$ denotes the four-momentum of the initial (final) quark, $P\left(P^{\prime}\right)$ stands for the four-momentum of $\mathcal{B}\left(\mathcal{B}^{\prime}\right)$ in the initial (final) state. For the case of a scalar diquark involved,

$$
\Gamma=\bar{\Gamma}^{\prime}=1,
$$

while for the case of an axial-vector diquark involved,

$$
\Gamma=-\frac{1}{\sqrt{3}} \gamma_{5} \phi^{*}\left(p_{2}, \lambda_{2}\right)
$$

and 


$$
\bar{\Gamma}^{\prime}=-\frac{1}{\sqrt{3}} \gamma_{5} \phi\left(p_{2}, \lambda_{2}\right)
$$

The transition matrix element $\left\langle\mathcal{B}^{\prime}\left(P^{\prime}, S_{z}^{\prime}\right)\left|\bar{s} \gamma_{\mu}\left(1-\gamma_{5}\right) b\right| \mathcal{B}\left(P, S_{z}\right)\right\rangle$ can be parametrized as

$$
\begin{gathered}
\left\langle\mathcal{B}^{\prime}\left(P^{\prime}, S_{z}^{\prime}\right)\left|\bar{s} \gamma_{\mu} b\right| \mathcal{B}\left(P, S_{z}\right)\right\rangle=\bar{u}\left(P^{\prime}, S_{z}^{\prime}\right)\left[\gamma_{\mu} f_{1}\left(q^{2}\right)+i \sigma_{\mu \nu} \frac{q^{\nu}}{M} f_{2}\left(q^{2}\right)+\frac{q_{\mu}}{M} f_{3}\left(q^{2}\right)\right] u\left(P, S_{z}\right), \\
\left\langle\mathcal{B}^{\prime}\left(P^{\prime}, S_{z}^{\prime}\right)\left|\bar{s} \gamma_{\mu} \gamma_{5} b\right| \mathcal{B}\left(P, S_{z}\right)\right\rangle=\bar{u}\left(P^{\prime}, S_{z}^{\prime}\right)\left[\gamma_{\mu} g_{1}\left(q^{2}\right)+i \sigma_{\mu \nu} \frac{q^{\nu}}{M} g_{2}\left(q^{2}\right)+\frac{q_{\mu}}{M} g_{3}\left(q^{2}\right)\right] \gamma_{5} u\left(P, S_{z}\right),
\end{gathered}
$$

while $\left\langle\mathcal{B}^{\prime}\left(P^{\prime}, S_{z}^{\prime}\right)\left|\bar{s} i \sigma_{\mu \nu} q^{\nu}\left(1+\gamma_{5}\right) b\right| \mathcal{B}\left(P, S_{z}\right)\right\rangle$ can be parametrized as

$$
\begin{aligned}
\left\langle\mathcal{B}^{\prime}\left(P^{\prime}, S_{z}^{\prime}\right)\left|\bar{s} i \sigma_{\mu \nu} \frac{q^{\nu}}{M} b\right| \mathcal{B}\left(P, S_{z}\right)\right\rangle & =\bar{u}\left(P^{\prime}, S_{z}^{\prime}\right)\left[\gamma_{\mu} f_{1}^{T}\left(q^{2}\right)+i \sigma_{\mu \nu} \frac{q^{\nu}}{M} f_{2}^{T}\left(q^{2}\right)+\frac{q_{\mu}}{M} f_{3}^{T}\left(q^{2}\right)\right] u\left(P, S_{z}\right), \\
\left\langle\mathcal{B}^{\prime}\left(P^{\prime}, S_{z}^{\prime}\right)\left|\bar{s} i \sigma_{\mu \nu} \frac{q^{\nu}}{M} \gamma_{5} b\right| \mathcal{B}\left(P, S_{z}\right)\right\rangle & =\bar{u}\left(P^{\prime}, S_{z}^{\prime}\right)\left[\gamma_{\mu} g_{1}^{T}\left(q^{2}\right)+i \sigma_{\mu \nu} \frac{q^{\nu}}{M} g_{2}^{T}\left(q^{2}\right)+\frac{q_{\mu}}{M} g_{3}^{T}\left(q^{2}\right)\right] \gamma_{5} u\left(P, S_{z}\right) .
\end{aligned}
$$

Here $q=P-P^{\prime}$, and $f_{i}^{(T)}, g_{i}^{(T)}$ are the form factors.

It should be noted that $f_{1}^{T}$ and $f_{3}^{T}$ are not independent. Multiply the first equation of Eq. (15) by $q^{\mu}$ to yield

$$
0=\bar{u}\left(P^{\prime}, S_{z}^{\prime}\right)\left[\left(M-M^{\prime}\right) f_{1}^{T}+\frac{q^{2}}{M} f_{3}^{T}\right] u\left(P, S_{z}\right)
$$

and one obtains

$$
f_{1}^{T}=-\frac{q^{2}}{M\left(M-M^{\prime}\right)} f_{3}^{T}
$$

In a similar way, one can obtain from the second equation of Eq. (15)

$$
g_{1}^{T}=\frac{q^{2}}{M\left(M+M^{\prime}\right)} g_{3}^{T}
$$

Taking the extraction of $f_{i}$ as an example, these form factors can be extracted as follows [49]. Multiplying the corresponding expressions in Eqs. (9) and (14) by $\bar{u}\left(P, S_{z}\right)\left(\Gamma^{\mu}\right)_{i} u\left(P^{\prime}, S_{z}^{\prime}\right)$ with $\left(\Gamma^{\mu}\right)_{i}=\left\{\gamma^{\mu}, P^{\mu}, P^{\prime \mu}\right\}$ respectively, and taking the approximation $P^{(\prime)} \rightarrow \bar{P}^{(\prime)}$ within the integral, and then summing over the polarizations in the initial and final states, one can arrive at

$$
\begin{aligned}
& \operatorname{Tr}\left\{\left(\Gamma^{\mu}\right)_{i}\left(\not P^{\prime}+M^{\prime}\right)\left(\gamma_{\mu} f_{1}+i \sigma_{\mu \nu} \frac{q^{\nu}}{M} f_{2}+\frac{q_{\mu}}{M} f_{3}\right)(\not P+M)\right\} \\
& =\int\left\{d^{3} p_{2}\right\} \frac{\varphi^{\prime}\left(x^{\prime}, k_{\perp}^{\prime}\right) \varphi\left(x, k_{\perp}\right)}{2 \sqrt{p_{1}^{+} p_{1}^{\prime+}\left(p_{1} \cdot \bar{P}+m_{1} M_{0}\right)\left(p_{1}^{\prime} \cdot \bar{P}^{\prime}+m_{1}^{\prime} M_{0}^{\prime}\right)}} \\
& \quad \times \sum_{\lambda_{2}} \operatorname{Tr}\left\{\left(\bar{\Gamma}^{\mu}\right)_{i}\left(\bar{P}^{\prime}+M_{0}^{\prime}\right) \bar{\Gamma}^{\prime}\left(\not p_{1}^{\prime}+m_{1}^{\prime}\right) \gamma_{\mu}\left(\not \not_{1}+m_{1}\right) \Gamma\left(\bar{P}+M_{0}\right)\right\}
\end{aligned}
$$

with $\left(\bar{\Gamma}^{\mu}\right)_{i}=\left\{\gamma^{\mu}, \bar{P}^{\mu}, \bar{P}^{\prime \mu}\right\}$. Then $f_{i}$ can be determined by solving linear equations. The form factors $g_{i}$ can be obtained in a similar way. Only $f_{2,3}^{T}$ or $g_{2,3}^{T}$ can be extracted in this way with $\left(\Gamma^{\mu}\right)_{i}=\left\{\gamma^{\mu}, P^{\mu}\right\}, f_{1}^{T}$ or $g_{1}^{T}$ is then obtained by Eq. (17) or (18).

\section{Flavor-spin wave functions}

In fact, the flavor-spin wave functions are not taken into account in the last subsection. This problem will be fixed in this subsection. We consider first the initial states.
For the doubly bottomed baryons, the wave functions are given as

$$
\mathcal{B}_{b b}=\frac{1}{\sqrt{2}}\left[\left(-\frac{\sqrt{3}}{2} b^{1}\left(b^{2} q\right)_{S}+\frac{1}{2} b^{1}\left(b^{2} q\right)_{A}\right)+\left(b^{1} \leftrightarrow b^{2}\right)\right],
$$

with $q=u, d$ or $s$ for $\Xi_{b b}^{0}, \Xi_{b b}^{-}$or $\Omega_{b b}^{-}$, respectively. For the bottom-charm baryons, there are two sets of states, as discussed in Sec. I. The wave functions of bottom-charm baryons with an axial-vector $b c$ diquark are given as 


$$
\mathcal{B}_{b c}=-\frac{\sqrt{3}}{2} b(c q)_{S}+\frac{1}{2} b(c q)_{A}
$$

while those with a scalar $b c$ diquark are

$$
\mathcal{B}_{b c}^{\prime}=-\frac{1}{2} b(c q)_{S}-\frac{\sqrt{3}}{2} b(c q)_{A}
$$

with $q=u, d$ or $s$ for $\Xi_{b c}^{(\prime)+}, \Xi_{b c}^{(\prime) 0}$ or $\Omega_{b c}^{(\prime) 0}$, respectively.

For the final states, the singly charmed baryon which belongs to antitriplets are given as

$$
\begin{aligned}
\Lambda_{c}^{+} & =-\frac{1}{2} d(c u)_{S}+\frac{\sqrt{3}}{2} d(c u)_{A}, \\
\Xi_{c}^{+} & =-\frac{1}{2} s(c u)_{S}+\frac{\sqrt{3}}{2} s(c u)_{A}, \\
\Xi_{c}^{0} & =-\frac{1}{2} s(c d)_{S}+\frac{\sqrt{3}}{2} s(c d)_{A}=\frac{1}{2} d(c s)_{S}-\frac{\sqrt{3}}{2} d(c s)_{A} .
\end{aligned}
$$

For the sextet of singly charmed baryons, the following wave functions are needed

$$
\begin{aligned}
\Sigma_{c}^{+} & =\frac{\sqrt{3}}{2} d(c u)_{S}+\frac{1}{2} d(c u)_{A}, \\
\Sigma_{c}^{0} & =\frac{1}{\sqrt{2}}\left[\frac{\sqrt{3}}{2} d^{1}\left(c d^{2}\right)_{S}+\frac{1}{2} d^{1}\left(c d^{2}\right)_{A}+\left(d^{1} \leftrightarrow d^{2}\right)\right], \\
\Xi_{c}^{\prime+} & =\frac{\sqrt{3}}{2} s(c u)_{S}+\frac{1}{2} s(c u)_{A}, \\
\Xi_{c}^{\prime 0} & =\frac{\sqrt{3}}{2} s(c d)_{S}+\frac{1}{2} s(c d)_{A}=\frac{\sqrt{3}}{2} d(c s)_{S}+\frac{1}{2} d(c s)_{A}, \\
\Omega_{c}^{0} & =\frac{1}{\sqrt{2}}\left[\frac{\sqrt{3}}{2} s^{1}\left(c s^{2}\right)_{S}+\frac{1}{2} s^{1}\left(c s^{2}\right)_{A}+\left(s^{1} \leftrightarrow s^{2}\right)\right] .
\end{aligned}
$$

The final states of singly bottomed baryons can be written down in a similar way.

Finally, the overlapping factors are determined by taking the inner product of the flavor-spin wave functions in the initial and final states. The corresponding results are collected in Table II for both $b \rightarrow s$ and $b \rightarrow d$ processes. The physical form factors are then obtained by

$$
F^{\text {phy }}=c_{S} F_{S}+c_{A} F_{A}
$$

where $F_{S(A)}$ denotes the form factor $f_{i}, g_{i}, f_{i}^{T}$, or $g_{i}^{T}$ with a scalar diquark (an axial-vector diquark) involved.

TABLE II. Flavor-spin space overlapping factors for $b \rightarrow s$ and $b \rightarrow d$ processes. Taking the $\Xi_{b b}^{0} \rightarrow \Xi_{b}^{0}$ as an example, the physical transition matrix elements can be evaluated as: $\left\langle\Xi_{b}^{0}\left|\Gamma_{\mu}\right| \Xi_{b b}^{0}\right\rangle=c_{S}\left\langle s[d i]\left|\Gamma_{\mu}\right| b[d i]\right\rangle+$ $c_{A}\left\langle s\{d i\}\left|\Gamma_{\mu}\right| b\{d i\}\right\rangle$ with $c_{S}=\sqrt{6} / 4$ and $c_{A}=\sqrt{6} / 4$. Here $[d i]$ and $\{d i\}$ denote a scalar and an axial-vector diquark, respectively.

\begin{tabular}{lccccc}
\hline \hline$b \rightarrow s$ process & $\left\langle s[d i]\left|\Gamma_{\mu}\right| b[d i]\right\rangle$ & $\left\langle s\{d i\}\left|\Gamma_{\mu}\right| b\{d i\}\right\rangle$ & $b \rightarrow d$ process & $\left\langle d[d i]\left|\Gamma_{\mu}\right| b[d i]\right\rangle$ & $\left\langle d\{d i\}\left|\Gamma_{\mu}\right| b\{d i\}\right\rangle$ \\
\hline$\Xi_{b b}^{0} \rightarrow \Xi_{b}^{0}$ & $\frac{\sqrt{6}}{4}$ & $\frac{\sqrt{6}}{4}$ & $\Xi_{b b}^{0} \rightarrow \Lambda_{b}^{0}$ & $\frac{\sqrt{6}}{4}$ & $\frac{\sqrt{6}}{4}$ \\
$\Xi_{b b}^{-} \rightarrow \Xi_{b}^{-}$ & $\frac{\sqrt{6}}{4}$ & $\frac{\sqrt{6}}{4}$ & $\Omega_{b b}^{-} \rightarrow \Xi_{b}^{-}$ & $-\frac{\sqrt{6}}{4}$ & $-\frac{\sqrt{6}}{4}$ \\
$\Xi_{b b}^{0} \rightarrow \Xi_{b}^{\prime 0}$ & $-\frac{3 \sqrt{2}}{4}$ & $\frac{\sqrt{2}}{4}$ & $\Xi_{b b}^{0} \rightarrow \Sigma_{b}^{0}$ & $-\frac{3 \sqrt{2}}{4}$ & $\frac{\sqrt{2}}{4}$ \\
$\Xi_{b b}^{-} \rightarrow \Xi_{b}^{\prime-}$ & $-\frac{3 \sqrt{2}}{4}$ & $\frac{\sqrt{2}}{4}$ & $\Xi_{b b}^{-} \rightarrow \Sigma_{b}^{-}$ & $-\frac{3}{2}$ \\
$\Omega_{b b}^{-} \rightarrow \Omega_{b}^{-}$ & $-\frac{3}{2}$ & $\frac{1}{2}$ & $\Omega_{b b}^{-} \rightarrow \Xi_{b}^{\prime-}$ & $-\frac{3 \sqrt{2}}{4}$ & $\frac{\sqrt{2}}{4}$ \\
$\Xi_{b c}^{+} \rightarrow \Xi_{c}^{+}$ & $\frac{\sqrt{3}}{4}$ & $\frac{\sqrt{3}}{4}$ & $\Xi_{b c}^{+} \rightarrow \Lambda_{c}^{+}$ & $\frac{\sqrt{3}}{4}$ & $\frac{\sqrt{3}}{4}$ \\
$\Xi_{b c}^{0} \rightarrow \Xi_{c}^{0}$ & $\frac{\sqrt{3}}{4}$ & $\frac{\sqrt{3}}{4}$ & $\Omega_{b c}^{0} \rightarrow \Xi_{c}^{0}$ & $-\frac{\sqrt{3}}{4}$ & $-\frac{\sqrt{3}}{4}$ \\
$\Xi_{b c}^{+} \rightarrow \Xi_{c}^{\prime+}$ & $-\frac{3}{4}$ & $\frac{1}{4}$ & $\Xi_{b c}^{+} \rightarrow \Sigma_{c}^{+}$ & $-\frac{3}{4}$ & $\frac{1}{4}$ \\
$\Xi_{b c}^{0} \rightarrow \Xi_{c}^{\prime 0}$ & $-\frac{3}{4}$ & $\frac{1}{4}$ & $\Xi_{b c}^{0} \rightarrow \Sigma_{c}^{0}$ & $-\frac{3 \sqrt{2}}{4}$ & $\frac{\sqrt{2}}{4}$ \\
$\Omega_{b c}^{0} \rightarrow \Omega_{c}^{0}$ & $-\frac{3 \sqrt{2}}{4}$ & $\frac{\sqrt{2}}{4}$ & $\Omega_{b c}^{0} \rightarrow \Xi_{c}^{\prime 0}$ & $-\frac{3}{4}$ & $\frac{1}{4}$ \\
$\Xi_{b c}^{\prime+} \rightarrow \Xi_{c}^{+}$ & $\frac{1}{4}$ & $-\frac{3}{4}$ & $\Xi_{b c}^{\prime+} \rightarrow \Lambda_{c}^{+}$ & $\frac{1}{4}$ & $-\frac{3}{4}$ \\
$\Xi_{b c}^{\prime 0} \rightarrow \Xi_{c}^{0}$ & $\frac{1}{4}$ & $-\frac{3}{4}$ & $\Omega_{b c}^{\prime 0} \rightarrow \Xi_{c}^{0}$ & $-\frac{1}{4}$ & $\frac{3}{4}$ \\
$\Xi_{b c}^{\prime+} \rightarrow \Xi_{c}^{\prime+}$ & $-\frac{\sqrt{3}}{4}$ & $-\frac{\sqrt{3}}{4}$ & $\Xi_{b c}^{\prime+} \rightarrow \Sigma_{c}^{+}$ & $-\frac{\sqrt{3}}{4}$ & $-\frac{\sqrt{3}}{4}$ \\
$\Xi_{b c}^{\prime 0} \rightarrow \Xi_{c}^{\prime 0}$ & $-\frac{\sqrt{3}}{4}$ & $-\frac{\sqrt{3}}{4}$ & $\Xi_{b c}^{\prime 0} \rightarrow \Sigma_{c}^{0}$ & $-\frac{\sqrt{6}}{4}$ & $-\frac{\sqrt{6}}{4}$ \\
$\Omega_{b c}^{\prime 0} \rightarrow \Omega_{c}^{0}$ & $-\frac{\sqrt{6}}{4}$ & $-\frac{\sqrt{6}}{4}$ & $\Omega_{b c}^{\prime 0} \rightarrow \Xi_{c}^{\prime 0}$ & $-\frac{\sqrt{3}}{4}$ & $-\frac{\sqrt{3}}{4}$ \\
\hline \hline
\end{tabular}




\section{Phenomenological observables}

The hadronic helicity amplitudes can be defined by

$$
\begin{aligned}
H_{\lambda^{\prime}, \lambda_{V}}^{V, \lambda} \equiv & \left(C_{9}^{\mathrm{eff}}\left(q^{2}\right)\left\langle\mathcal{B}^{\prime}\left|\bar{s} \gamma^{\mu}\left(1-\gamma_{5}\right) b\right| \mathcal{B}\right\rangle\right. \\
& \left.-C_{7}^{\mathrm{eff}} 2 m_{b}\left\langle\mathcal{B}^{\prime}\left|\bar{s} i \sigma^{\mu \nu} \frac{q_{\nu}}{q^{2}}\left(1+\gamma_{5}\right) b\right| \mathcal{B}\right\rangle\right) \epsilon_{\mu}^{*}\left(\lambda_{V}\right), \\
H_{\lambda^{\prime}, t}^{V, \lambda} \equiv & \left(C_{9}^{\mathrm{eff}}\left(q^{2}\right)\left\langle\mathcal{B}^{\prime}\left|\bar{s} \gamma^{\mu}\left(1-\gamma_{5}\right) b\right| \mathcal{B}\right\rangle\right) \frac{q_{\mu}}{\sqrt{q^{2}}},
\end{aligned}
$$

and

$$
\begin{aligned}
H_{\lambda^{\prime}, \lambda_{V}}^{A, \lambda} & \equiv\left(C_{10}\left\langle\mathcal{B}^{\prime}\left|\bar{s} \gamma^{\mu}\left(1-\gamma_{5}\right) b\right| \mathcal{B}\right\rangle\right) \epsilon_{\mu}^{*}\left(\lambda_{V}\right), \\
H_{\lambda^{\prime}, t}^{A, \lambda} & \equiv\left(C_{10}\left\langle\mathcal{B}^{\prime}\left|\bar{s} \gamma^{\mu}\left(1-\gamma_{5}\right) b\right| \mathcal{B}\right\rangle\right) \frac{q_{\mu}}{\sqrt{q^{2}}},
\end{aligned}
$$

where $\epsilon_{\mu}\left(q_{\mu}\right)$ is the polarization vector (four-momentum) for an intermediate vector particle, $\lambda_{V}$ denotes its polarization, $\lambda^{(\prime)}$ is the polarization of the baryon in the initial (final) state. Hereafter the superscript " $V$ " (" $A$ ") always means that its corresponding leptonic counterpart is $\bar{l} \gamma^{\mu} l$ $\left(\bar{l} \gamma^{\mu} \gamma_{5} l\right)$. It should not be confused with the notation of the vector current (axial-vector current) in the hadronic matrix element.

Note that Eqs. (14) and (15) have the same parametrization, so it is convenient to introduce the following notations:

$$
\begin{aligned}
& F_{i}^{V}\left(q^{2}\right) \equiv C_{9}^{\mathrm{eff}}\left(q^{2}\right) f_{i}\left(q^{2}\right)-C_{7}^{\mathrm{eff}} \frac{2 m_{b} M}{q^{2}} f_{i}^{T}\left(q^{2}\right), \\
& G_{i}^{V}\left(q^{2}\right) \equiv C_{9}^{\mathrm{eff}}\left(q^{2}\right) g_{i}\left(q^{2}\right)+C_{7}^{\mathrm{eff}} \frac{2 m_{b} M}{q^{2}} g_{i}^{T}\left(q^{2}\right)
\end{aligned}
$$

and

$$
\begin{aligned}
F_{i}^{A}\left(q^{2}\right) & \equiv C_{10} f_{i}\left(q^{2}\right), \\
G_{i}^{A}\left(q^{2}\right) & \equiv C_{10} g_{i}\left(q^{2}\right) .
\end{aligned}
$$

Then the $\Gamma^{\mu}$ and $\Gamma^{\mu} \gamma_{5}$ parts in Eq. (26) are calculated respectively as:

$$
\begin{gathered}
H V_{\frac{1}{2}, 0}^{V,-\frac{1}{2}}=-i \frac{\sqrt{Q_{-}}}{\sqrt{q^{2}}}\left(\left(M+M^{\prime}\right) F_{1}^{V}-\frac{q^{2}}{M} F_{2}^{V}\right), \\
H V_{\frac{1}{2}, 1}^{V, \frac{1}{2}}=i \sqrt{2 Q_{-}}\left(-F_{1}^{V}+\frac{M+M^{\prime}}{M} F_{2}^{V}\right), \\
H A_{\frac{1}{2}, 0}^{V,-\frac{1}{2}}=-i \frac{\sqrt{Q_{+}}}{\sqrt{q^{2}}}\left(\left(M-M^{\prime}\right) G_{1}^{V}+\frac{q^{2}}{M} G_{2}^{V}\right), \\
H A_{\frac{1}{2}, 1}^{V, \frac{1}{2}}=i \sqrt{2 Q_{+}}\left(-G_{1}^{V}-\frac{M-M^{\prime}}{M} G_{2}^{V}\right)
\end{gathered}
$$

and

$$
\begin{aligned}
& H V_{-\lambda^{\prime},-\lambda_{V}}^{V,-\lambda}=H V_{\lambda^{\prime}, \lambda_{V}}^{V, \lambda}, \\
& H A_{-\lambda^{\prime},-\lambda_{V}}^{V,-\lambda}=-H A_{\lambda^{\prime}, \lambda_{V}}^{V, \lambda} .
\end{aligned}
$$

The total hadronic helicity amplitude is then given by

$$
H_{\lambda^{\prime}, \lambda_{V}}^{V, \lambda}=H V_{\lambda^{\prime}, \lambda_{V}}^{V, \lambda}-H A_{\lambda^{\prime}, \lambda_{V}}^{V, \lambda}
$$

$H_{\lambda^{\prime}, \lambda_{V}}^{A, \lambda}$ has the complete the same form as the corresponding $H_{\lambda^{\prime}, \lambda_{V}}^{V, \lambda}$ but with the following replacements:

$$
\begin{aligned}
F_{i}^{V} & \rightarrow F_{i}^{A}, \\
G_{i}^{V} & \rightarrow G_{i}^{A} .
\end{aligned}
$$

In addition, the timelike polarizations for $H^{A}$ are also needed

$$
\begin{gathered}
H V_{-\frac{1}{2}, t}^{A, \frac{1}{2}}=H V_{\frac{1}{2}, t}^{A,-\frac{1}{2}}=-i \frac{\sqrt{Q_{+}}}{\sqrt{q^{2}}}\left(\left(M-M^{\prime}\right) F_{1}^{A}+\frac{q^{2}}{M} F_{3}^{A}\right), \\
-H A_{-\frac{1}{2}, t}^{A, \frac{1}{2}}=H A_{\frac{1}{2}, t}^{A,-\frac{1}{2}}=-i \frac{\sqrt{Q_{-}}}{\sqrt{q^{2}}}\left(\left(M+M^{\prime}\right) G_{1}^{A}-\frac{q^{2}}{M} G_{3}^{A}\right)
\end{gathered}
$$

and

$$
H_{\lambda^{\prime}, t}^{A, \lambda}=H V_{\lambda^{\prime}, t}^{A, \lambda}-H A_{\lambda^{\prime}, t}^{A, \lambda} .
$$

Finally, the angular distribution is given by the following expression

$$
\frac{d^{2} \Gamma}{d q^{2} d \cos \theta}=\frac{\left|\vec{P}^{\prime}\right|\left|\vec{p}_{1}\right|}{16(2 \pi)^{3} M^{2} \sqrt{q^{2}}} \overline{|\mathcal{M}|^{2}}
$$

Here the squared amplitude is

$$
\overline{|\mathcal{M}|^{2}}=\frac{1}{2}|\lambda|^{2}\left(I_{0}+I_{1} \cos \theta+I_{2} \cos 2 \theta\right)
$$

with

$$
\lambda \equiv \frac{G_{F}}{\sqrt{2}} V_{t b} V_{t s}^{*} \frac{\alpha_{\mathrm{em}}}{2 \pi}
$$

and 


$$
\begin{aligned}
I_{0}= & \left(q^{2}+4 m_{l}^{2}\right)\left(\left|H_{-\frac{1}{2}, 0}^{V, \frac{1}{2}}\right|^{2}+\left|H_{\frac{1}{2}, 0}^{V,-\frac{1}{2}}\right|^{2}\right)+\left(\frac{3}{2} q^{2}+2 m_{l}^{2}\right)\left(\left|H_{\frac{1}{2}, 1}^{V, \frac{1}{2}}\right|^{2}+\left|H_{-\frac{1}{2},-1}^{V,-\frac{1}{2}}\right|^{2}\right) \\
& +\left(q^{2}-4 m_{l}^{2}\right)\left(\frac{3}{2}\left|H_{\frac{1}{2}, 1}^{A, \frac{1}{2}}\right|^{2}+\frac{3}{2}\left|H_{-\frac{1}{2},-1}^{A,-\frac{1}{2}}\right|^{2}+\left|H_{-\frac{1}{2}, 0}^{A, \frac{1}{2}}\right|^{2}+\left|H_{\frac{1}{2}, 0}^{A,-\frac{1}{2}}\right|^{2}\right)+8 m_{l}^{2}\left(\left|H_{-\frac{1}{2}, t}^{A, \frac{1}{2}}\right|^{2}+\left|H_{\frac{1}{2}, t}^{A,-\frac{1}{2}}\right|^{2}\right), \\
I_{1}= & 4 \sqrt{q^{2}\left(q^{2}-4 m_{l}^{2}\right)} \operatorname{Re}\left(H_{\frac{1}{2}, 1}^{A, \frac{1}{2} *} H_{\frac{1}{2}, 1}^{V, \frac{1}{2}}-H_{-\frac{1}{2},-1}^{A,-\frac{1}{2} *} H_{-\frac{1}{2},-1}^{V,-\frac{1}{2}}\right), \\
I_{2}= & \frac{1}{2}\left(q^{2}-4 m_{l}^{2}\right)\left(\left|H_{\frac{1}{2}, 1}^{V, \frac{1}{2}}\right|^{2}+\left|H_{-\frac{1}{2},-1}^{V,-\frac{1}{2}}\right|^{2}-2\left|H_{-\frac{1}{2}, 0}^{V, \frac{1}{2}}\right|^{2}-2\left|H_{\frac{1}{2}, 0}^{V,-\frac{1}{2}}\right|^{2}+\left|H_{\frac{1}{2}, 1}^{A, \frac{1}{2}}\right|^{2}+\left|H_{-\frac{1}{2},-1}^{A,-\frac{1}{2}}\right|^{2}-2\left|H_{-\frac{1}{2}, 0}^{A, \frac{1}{2}}\right|^{2}-2\left|H_{\frac{1}{2}, 0}^{A,-\frac{1}{2}}\right|^{2}\right) .
\end{aligned}
$$

The differential decay width is given as

$$
\frac{d \Gamma}{d q^{2}}=\frac{d \Gamma_{L}}{d q^{2}}+\frac{d \Gamma_{T}}{d q^{2}}
$$

where the $q^{2}$ is the invariant mass of the dilepton and the longitudinally and transversely polarized decay widths are respectively

$$
\begin{aligned}
\frac{d \Gamma_{L}}{d q^{2}}= & |\lambda|^{2} \frac{\left|\vec{P}^{\prime}\right|\left|\vec{p}_{1}\right|}{12(2 \pi)^{3} M^{2} \sqrt{q^{2}}}\left\{\left(q^{2}+2 m_{l}^{2}\right)\left(\left|H_{-\frac{1}{2}, 0}^{V, \frac{1}{2}}\right|^{2}+\left|H_{\frac{1}{2}, 0}^{V,-\frac{1}{2}}\right|^{2}\right)+\left(q^{2}-4 m_{l}^{2}\right)\left(\left|H_{-\frac{1}{2}, 0}^{A, \frac{1}{2}}\right|^{2}+\left|H_{\frac{1}{2}, 0}^{A,-\frac{1}{2}}\right|^{2}\right)\right. \\
& \left.+6 m_{l}^{2}\left(\left|H_{-\frac{1}{2}, t}^{A, \frac{1}{2}}\right|^{2}+\left|H_{\frac{1}{2}, t}^{A,-\frac{1}{2}}\right|^{2}\right)\right\}, \\
\frac{d \Gamma_{T}}{d q^{2}}= & |\lambda|^{2} \frac{\left|\vec{P}^{\prime}\right|\left|\vec{p}_{1}\right|}{12(2 \pi)^{3} M^{2} \sqrt{q^{2}}}\left\{\left(q^{2}+2 m_{l}^{2}\right)\left(\left|H_{\frac{1}{2}, 1}^{V, \frac{1}{2}}\right|^{2}+\left|H_{-\frac{1}{2},-1}^{V,-\frac{1}{2}}\right|^{2}\right)+\left(q^{2}-4 m_{l}^{2}\right)\left(\left|H_{\frac{1}{2}, 1}^{A, \frac{1}{2}}\right|^{2}+\left|H_{-\frac{1}{2},-1}^{A,-\frac{1}{2}}\right|^{2}\right)\right\} .
\end{aligned}
$$

The normalized differential forward-backward asymmetry is defined by

$$
\frac{d \bar{A}_{F B}}{d q^{2}} \equiv \frac{\left(\int_{0}^{1}-\int_{-1}^{0}\right) d \cos \theta \frac{d^{2} \Gamma}{d q^{2} d \cos \theta}}{\left(\int_{0}^{1}+\int_{-1}^{0}\right) d \cos \theta \frac{d^{2} \Gamma}{d q^{2} d \cos \theta}} .
$$

Then one can obtain

$$
\frac{d \bar{A}_{F B}}{d q^{2}}=\frac{I_{1}}{2\left(I_{0}-I_{2} / 3\right)}
$$

when substituting Eqs. (36) and (37) into Eq. (43).

\section{NUMERICAL RESULTS AND DISCUSSIONS}

\section{A. Inputs}

The constituent quark masses are given as (in units of $\mathrm{GeV}$ ) [36-44]

\begin{tabular}{|c|c|c|c|c|c|c|}
\hline Baryons & $\Xi_{b c}^{(\prime)+}$ & $\Xi_{b c}^{(\prime) 0}$ & $\Omega_{b c}^{(\prime) 0}$ & $\Xi_{b b}^{0}$ & $\Xi_{b b}^{-}$ & $\Omega_{b b}^{-}$ \\
\hline Masses & $6.943[26]$ & $6.943[26]$ & $6.998[26]$ & $10.143[26]$ & 10.143 [26] & $10.273[26]$ \\
\hline Lifetimes & $244[55]$ & $93[55]$ & $220[56]$ & $370[55]$ & 370 [55] & $800[56]$ \\
\hline
\end{tabular}

$$
m_{u}=m_{d}=0.25, \quad m_{s}=0.37, \quad m_{c}=1.4, \quad m_{b}=4.8 .
$$

The masses of the scalar and axial-vector diquarks are approximated by $m_{[Q q]}=m_{\{Q q\}}=m_{Q}+m_{q}$. The shape parameters $\beta$ in Eq. (8) are given as (in units of $\mathrm{GeV}$ ) [31]

$$
\begin{aligned}
& \beta_{d[c q]}=\beta_{d\{c q\}}=0.470, \quad \beta_{s[c q]}=\beta_{s\{c q\}}=0.535, \quad \beta_{b[c q]}=\beta_{b\{c q\}}=0.886, \\
& \beta_{d[b q]}=\beta_{d\{b q\}}=0.562, \quad \beta_{s[b q]}=\beta_{s\{b q\}}=0.623, \quad \beta_{b[b q]}=\beta_{b\{b q\}}=1.472,
\end{aligned}
$$

TABLE III. Masses (in units of $\mathrm{GeV}$ ) and lifetimes (in units of fs) of doubly heavy baryons. We have quoted the results from Refs. $[26,55,56]$. 
TABLE IV. Masses (in units of $\mathrm{GeV}$ ) of baryons in the final states [57].

\begin{tabular}{lccccccc}
\hline \hline$\Lambda_{c}^{+}$ & $\Xi_{c}^{+}$ & $\Xi_{c}^{0}$ & $\Sigma_{c}^{+}$ & $\Sigma_{c}^{0}$ & $\Xi_{c}^{\prime+}$ & $\Xi_{c}^{\prime 0}$ & $\Omega_{c}^{0}$ \\
\hline 2.286 & 2.468 & 2.471 & 2.453 & 2.454 & 2.576 & 2.578 & 2.695 \\
\hline \hline$\Lambda_{b}^{0}$ & $\Xi_{b}^{0}$ & $\Xi_{b}^{-}$ & $\Sigma_{b}^{0}$ & $\Sigma_{b}^{-}$ & $\Xi_{b}^{\prime 0}$ & $\Xi_{b}^{\prime-}$ & $\Omega_{b}^{-}$ \\
\hline 5.620 & 5.793 & 5.795 & 5.814 & 5.816 & 5.935 & 5.935 & 6.046 \\
\hline \hline
\end{tabular}

where $q=u, d, s$.

The masses and lifetimes of the parent baryons are collected in Table III $[26,55,56]$. The masses of the daughter baryons are given in Table IV [57]. Fermi constant and CKM matrix elements are give as [57]

$$
\begin{aligned}
G_{F} & =1.166 \times 10^{-5} \mathrm{GeV}^{-2}, \\
\left|V_{t b}\right| & =0.999, \quad\left|V_{t s}\right|=0.0403, \quad\left|V_{t d}\right|=0.00875 .
\end{aligned}
$$

\section{B. Results for form factors}

To access the $q^{2}$-distribution, the following single pole structure is assumed for form factors

$$
F\left(q^{2}\right)=\frac{F(0)}{1-\frac{q^{2}}{m_{\mathrm{pole}}^{2}}} .
$$

Here $F(0)$ is the value of the form factor at $q^{2}=0$, and the numerical results for $f_{i}^{(T)}$ and $g_{i}^{(T)}$ predicted by the lightfront approach are collected in Tables V-VIII for $b \rightarrow s$ process and Tables IX-XII for $b \rightarrow d$ process. $m_{\text {pole }}$ is taken as $5.37 \mathrm{GeV}$ for $b \rightarrow s$ process and $5.28 \mathrm{GeV}$ for $b \rightarrow d$ process, which, in practice, are taken as the masses of $B_{s}$ and $B$ mesons, respectively. The discussion for the validity of this assumption can be found in our previous work [44].

The physical form factors can then be obtained by Eqs. (25) and (48).

TABLE V. Values of form factors $f_{i}$ and $g_{i}$ at $q^{2}=0$ for $b \rightarrow s$ process in $b b$ sector. The left (right) half of the table corresponds to a scalar diquark (an axial-vector diquark) involved case.

\begin{tabular}{lccccccc}
\hline \hline$F$ & $F(0)$ & $F$ & $F(0)$ & $F$ & $F(0)$ & $F$ & $F(0)$ \\
\hline$f_{1, S}^{\Xi_{b b} \rightarrow \Xi_{b}}$ & 0.141 & $g_{1, S}^{\Xi_{b b} \rightarrow \Xi_{b}}$ & 0.122 & $f_{1, A}^{\Xi_{b b} \rightarrow \Xi_{b}}$ & 0.138 & $g_{1, A}^{\Xi_{b b} \rightarrow \Xi_{b}}$ & -0.030 \\
$f_{2, S}^{\Xi_{b b} \rightarrow \Xi_{b}}$ & -0.189 & $g_{2, S}^{\Xi_{b b} \rightarrow \Xi_{b}}$ & 0.056 & $f_{2, A}^{\Xi_{b b} \rightarrow \Xi_{b}}$ & 0.132 & $g_{2, A}^{\Xi_{b b} \rightarrow \Xi_{b}}$ & -0.055 \\
$f_{3, S}^{\Xi_{b b} \rightarrow \Xi_{b}}$ & 0.016 & $g_{3, S}^{\Xi_{b b} \rightarrow \Xi_{b}}$ & -0.406 & $f_{3, A}^{\Xi_{b b} \rightarrow \Xi_{b}}$ & -0.068 & $g_{3, A}^{\Xi_{b b} \rightarrow \Xi_{b}}$ & 0.261 \\
$f_{1, S}^{\Xi_{b b} \rightarrow \Xi_{b}^{\prime}}$ & 0.143 & $g_{1, S}^{\Xi_{b b} \rightarrow \Xi_{b}^{\prime}}$ & 0.130 & $f_{1, A}^{\Xi_{b b} \rightarrow \Xi_{b}^{\prime}}$ & 0.140 & $g_{1, A}^{\Xi_{b b} \rightarrow \Xi_{b}^{\prime}}$ & -0.031 \\
$f_{2, S}^{\Xi_{b b} \rightarrow \Xi_{b}^{\prime}}$ & -0.202 & $g_{2, S}^{\Xi_{b b} \rightarrow \Xi_{b}^{\prime}}$ & 0.024 & $f_{2, A}^{\Xi_{b b} \rightarrow \Xi_{b}^{\prime}}$ & 0.138 & $g_{2, A} \rightarrow \Xi_{b}^{\prime}$ & -0.048 \\
$f_{3, S}^{\Xi_{b b} \rightarrow \Xi_{b}^{\prime}}$ & 0.003 & $g_{3, S}^{\Xi_{b b} \rightarrow \Xi_{b}^{\prime}}$ & -0.316 & $f_{3, A}^{\Xi_{b b} \rightarrow \Xi_{b}^{\prime}}$ & -0.082 & $g_{3, A}^{\Xi_{b b} \rightarrow \Xi_{b}^{\prime}}$ & 0.249 \\
$f_{1, S}^{\Omega_{b b}^{-} \rightarrow \Omega_{b}^{-}}$ & 0.139 & $g_{1, S}^{\Omega_{b b}^{-} \rightarrow \Omega_{b}^{-}}$ & 0.125 & $f_{1, A}^{\Omega_{b b}^{-} \rightarrow \Omega_{b}^{-}}$ & 0.136 & $\Omega_{1, A}^{-} \rightarrow \Omega_{b}^{-}$ & -0.030 \\
$f_{2, S}^{\Omega_{b b}^{-} \rightarrow \Omega_{b}^{-}}$ & -0.198 & $g_{b b}^{-} \rightarrow \Omega_{b}^{-}$ & 0.028 & $f_{2, A}^{\Omega_{b b}^{-} \rightarrow \Omega_{b}^{-}}$ & 0.134 & $g_{b, A}^{-} \rightarrow \Omega_{b}^{-}$ & -0.048 \\
$f_{3, S}^{\Omega_{b b}^{-} \rightarrow \Omega_{b}^{-}}$ & 0.003 & $g_{3, S}^{-} \rightarrow \Omega_{b}^{-}$ & -0.332 & $f_{3, A}^{\Omega_{b b}^{-} \rightarrow \Omega_{b}^{-}}$ & -0.079 & $\Omega_{3, A}^{-} \rightarrow \Omega_{b}^{-}$ & 0.250 \\
\hline \hline
\end{tabular}

TABLE VI. Values of form factors $f_{i}^{T}$ and $g_{i}^{T}$ at $q^{2}=0$ for $b \rightarrow s$ process in $b b$ sector. The left (right) half of the table corresponds to a scalar diquark (an axial-vector diquark) involved case. $f_{1}^{T}$ and $g_{1}^{T}$ are obtained by Eqs. (17) and (18) respectively.

\begin{tabular}{lccccrrr}
\hline \hline$F$ & $F(0)$ & $F$ & $F(0)$ & $F$ & $F(0)$ & \multicolumn{1}{c}{$F$} & $F(0)$ \\
\hline$f_{2, S}^{T, \Xi_{b b} \rightarrow \Xi_{b}}$ & 0.108 & $g_{2, S}^{T, \Xi_{b b} \rightarrow \Xi_{b}}$ & 0.128 & $f_{2, A}^{T, \Xi_{b b} \rightarrow \Xi_{b}}$ & -0.066 & $g_{2, A}^{T, \Xi_{b b} \rightarrow \Xi_{b}}$ & -0.049 \\
$f_{3, S}^{T, \Xi_{b b} \rightarrow \Xi_{b}}$ & 0.091 & $g_{3, S}^{T, \Xi_{b} \rightarrow \Xi_{b}}$ & 0.156 & $f_{3, A}^{T, \Xi_{b b} \rightarrow \Xi_{b}}$ & 0.134 & $g_{3, A}^{T, \Xi_{b b} \rightarrow \Xi_{b}}$ & 0.032 \\
$f_{2, S}^{T, \Xi_{b b} \rightarrow \Xi_{b}^{\prime}}$ & 0.117 & $g_{2, S}^{T, \Xi_{b b} \rightarrow \Xi_{b}^{\prime}}$ & 0.127 & $f_{2, A}^{T, \Xi_{b b} \rightarrow \Xi_{b}^{\prime}}$ & -0.068 & $g_{2, A}^{T, \Xi_{b b} \rightarrow \Xi_{b}^{\prime}}$ & -0.049 \\
$f_{3, S}^{T, \Xi_{b b} \rightarrow \Xi_{b}^{\prime}}$ & 0.091 & $g_{3, S}^{T, \Xi_{b} \rightarrow \Xi_{b}^{\prime}}$ & 0.198 & $f_{3, A}^{T, \Xi_{b b} \rightarrow \Xi_{b}^{\prime}}$ & 0.134 & $g_{3, A}^{T, \Xi_{b b} \rightarrow \Xi_{b}^{\prime}}$ & 0.026 \\
$f_{2, S}^{T, \Omega_{b}^{-} \rightarrow \Omega_{b}^{-}}$ & 0.112 & $g_{2, S}^{T, \Omega_{b}^{-} \rightarrow \Omega_{b}^{-}}$ & 0.123 & $f_{2, A}^{T, \Omega_{b b}^{-} \rightarrow \Omega_{b}^{-}}$ & -0.065 & $g_{2, A}^{T, \Omega_{b b}^{-} \rightarrow \Omega_{b}^{-}}$ & -0.047 \\
$f_{3, S}^{T, \Omega_{b b}^{-} \rightarrow \Omega_{b}^{-}}$ & 0.088 & $g_{3, S}^{T, \Omega_{b}^{-} \rightarrow \Omega_{b}^{-}}$ & 0.186 & $f_{3, A}^{T, \Omega_{b}^{-} \rightarrow \Omega_{b}^{-}}$ & 0.130 & $g_{3, A}^{T, \Omega_{b b}^{-} \rightarrow \Omega_{b}^{-}}$ & 0.027 \\
\hline \hline
\end{tabular}


TABLE VII. Same as Table V but for $b \rightarrow s$ process in $b c$ sector. $b c^{\prime}$ sector has the same form factors.

\begin{tabular}{lccccccc}
\hline \hline$F$ & $F(0)$ & $F$ & $F(0)$ & $F$ & $F(0)$ & $F$ & $F(0)$ \\
\hline$f_{1, S}^{\Xi_{b c} \rightarrow \Xi_{c}}$ & 0.203 & $g_{1, S}^{\Xi_{b c} \rightarrow \Xi_{c}}$ & 0.167 & $f_{1, A}^{\Xi_{b c} \rightarrow \Xi_{c}}$ & 0.185 & $g_{1, A}^{\Xi_{b c} \rightarrow \Xi_{c}}$ & -0.033 \\
$f_{2, S}^{\Xi_{b c} \rightarrow \Xi_{c}}$ & -0.079 & $g_{2, S}^{\Xi_{b c} \rightarrow \Xi_{c}}$ & 0.097 & $f_{2, A}^{\Xi_{b c} \rightarrow \Xi_{c}}$ & 0.203 & $g_{2, A}^{\Xi_{b c} \rightarrow \Xi_{c}}$ & -0.068 \\
$f_{3, S}^{\Xi_{b c} \rightarrow \Xi_{c}}$ & 0.015 & $g_{3, S}^{\Xi_{b c} \rightarrow \Xi_{c}}$ & -0.329 & $f_{3, A}^{\Xi_{b c} \rightarrow \Xi_{c}}$ & -0.109 & $g_{3, A}^{\Xi_{b c} \rightarrow \Xi_{c}}$ & 0.166 \\
$f_{1, S}^{\Xi_{b c} \rightarrow \Xi_{c}^{\prime}}$ & 0.204 & $g_{1, S}^{\Xi_{b c} \rightarrow \Xi_{c}^{\prime}}$ & 0.174 & $f_{1, A}^{\Xi_{b c} \rightarrow \Xi_{c}^{\prime}}$ & 0.186 & $g_{1, A}^{\Xi_{b c} \rightarrow \Xi_{c}^{\prime}}$ & -0.035 \\
$f_{2, S}^{\Xi_{b c} \rightarrow \Xi_{c}^{\prime}}$ & -0.090 & $g_{2, S}^{\Xi_{b c} \rightarrow \Xi_{c}^{\prime}}$ & 0.074 & $f_{2, A}^{\Xi_{b c} \rightarrow \Xi_{c}^{\prime}}$ & 0.205 & $g_{2, A}^{\Xi_{b c} \rightarrow \Xi_{c}^{\prime}}$ & -0.063 \\
$f_{3, S}^{\Xi_{b c} \rightarrow \Xi_{c}^{\prime}}$ & 0.007 & $g_{3, S}^{\Xi_{b c} \rightarrow \Xi_{c}^{\prime}}$ & -0.300 & $f_{3, A}^{\Xi_{b c} \rightarrow \Xi_{c}^{\prime}}$ & -0.116 & $g_{3, A}^{\Xi_{b c} \rightarrow \Xi_{c}^{\prime}}$ & 0.164 \\
$f_{1, S}^{\Omega_{b c}^{0} \rightarrow \Omega_{c}^{0}}$ & 0.192 & $g_{1, S}^{\Omega_{b c}^{0} \rightarrow \Omega_{c}^{0}}$ & 0.165 & $f_{1, A}^{\Omega_{b c}^{0} \rightarrow \Omega_{c}^{0}}$ & 0.177 & $g_{1, A}^{\Omega_{b c}^{0} \rightarrow \Omega_{c}^{0}}$ & -0.033 \\
$f_{2, S}^{\Omega_{b c}^{0} \rightarrow \Omega_{c}^{0}}$ & -0.091 & $g_{2, S}^{\Omega_{b c}^{0} \rightarrow \Omega_{c}^{0}}$ & 0.064 & $f_{2, A}^{\Omega_{b c}^{0} \rightarrow \Omega_{c}^{0}}$ & 0.194 & $g_{2, A}^{0} \rightarrow \Omega_{c}^{0}$ & -0.061 \\
$f_{3, S}^{\Omega_{b c}^{0} \rightarrow \Omega_{c}^{0}}$ & 0.004 & $g_{3, S}^{0} \rightarrow \Omega_{c}^{0}$ & -0.288 & $f_{3, A}^{\Omega_{b c}^{0} \rightarrow \Omega_{c}^{0}}$ & -0.112 & $\Omega_{b, A}^{0} \rightarrow \Omega_{c}^{0}$ & 0.163 \\
\hline \hline
\end{tabular}

TABLE VIII. Same as Table VI but for $b \rightarrow s$ process in $b c$ sector. $b c^{\prime}$ sector has the same form factors.

\begin{tabular}{lccccccc}
\hline \hline$F$ & $F(0)$ & $F$ & $F(0)$ & $F$ & $F(0)$ & $F$ & $F(0)$ \\
\hline$f_{2, S}^{T, \Xi_{b c} \rightarrow \Xi_{c}}$ & 0.160 & $g_{2, S}^{T, \Xi_{b c} \rightarrow \Xi_{c}}$ & 0.202 & $f_{2, A}^{T, \Xi_{b c} \rightarrow \Xi_{c}}$ & -0.070 & $g_{2, A}^{T, \Xi_{b c} \rightarrow \Xi_{c}}$ & -0.072 \\
$f_{3, S}^{T, \Xi_{b c} \rightarrow \Xi_{c}}$ & 0.085 & $g_{3, S}^{T, \Xi_{b c} \rightarrow \Xi_{c}}$ & -0.021 & $f_{3, A}^{T, \Xi_{b c} \rightarrow \Xi_{c}}$ & 0.172 & $g_{3, A}^{T, \Xi_{b c} \rightarrow \Xi_{c}}$ & 0.068 \\
$f_{2, S}^{T, \Xi_{b c} \rightarrow \Xi_{c}^{\prime}}$ & 0.169 & $g_{2, S}^{T, \Xi_{b c} \rightarrow \Xi_{c}^{\prime}}$ & 0.200 & $f_{2, A}^{T, \Xi_{b c} \rightarrow \Xi_{c}^{\prime}}$ & -0.071 & $g_{2, A}^{T, \Xi_{b c} \rightarrow \Xi_{c}^{\prime}}$ & -0.072 \\
$f_{3, S}^{T, \Xi_{b c} \rightarrow \Xi_{c}^{\prime}}$ & 0.083 & $g_{3, S}^{T, \Xi_{b c} \rightarrow \Xi_{c}^{\prime}}$ & -0.006 & $f_{3, A}^{T, \Xi_{b c} \rightarrow \Xi_{c}^{\prime}}$ & 0.170 & $g_{3, A}^{T, \Xi_{b c} \rightarrow \Xi_{c}^{\prime}}$ & 0.068 \\
$f_{2, S}^{T, \Omega_{b c}^{0} \rightarrow \Omega_{c}^{0}}$ & 0.159 & $g_{2, S}^{T, \Omega_{b c}^{0} \rightarrow \Omega_{c}^{0}}$ & 0.188 & $f_{2, A}^{T, \Omega_{b c}^{0} \rightarrow \Omega_{c}^{0}}$ & -0.070 & $g_{2, A}^{T, \Omega_{b c}^{0} \rightarrow \Omega_{c}^{0}}$ & -0.069 \\
$f_{3, S}^{T, \Omega_{b c}^{0} \rightarrow \Omega_{c}^{0}}$ & 0.081 & $g_{3, S}^{T, \Omega_{b c}^{0} \rightarrow \Omega_{c}^{0}}$ & -0.001 & $f_{3, A}^{T, \Omega_{b c}^{0} \rightarrow \Omega_{c}^{0}}$ & 0.163 & $g_{3, A}^{T, \Omega_{b c}^{0} \rightarrow \Omega_{c}^{0}}$ & 0.067 \\
\hline \hline
\end{tabular}

TABLE IX. Same as Table V but for $b \rightarrow d$ process.

\begin{tabular}{|c|c|c|c|c|c|c|c|}
\hline$F$ & $F(0)$ & $F$ & $F(0)$ & $F$ & $F(0)$ & $F$ & $F(0)$ \\
\hline$f_{1, S}^{\Xi_{b b}^{0} \rightarrow \Lambda_{b}^{0}}$ & 0.100 & $g_{1, S}^{\Xi_{b b}^{0} \rightarrow \Lambda_{b}^{0}}$ & 0.087 & $f_{1, A}^{\Xi_{b b}^{0} \rightarrow \Lambda_{b}^{0}}$ & 0.098 & $g_{1, A}^{\Xi_{b b}^{0} \rightarrow \Lambda_{b}^{0}}$ & -0.020 \\
\hline$f_{2, S}^{\Xi_{b b}^{0} \rightarrow \Lambda_{b}^{0}}$ & -0.136 & $g_{2, S}^{\Xi_{b b}^{0} \rightarrow \Lambda_{b}^{0}}$ & 0.041 & $f_{2, A}^{\Xi_{b b}^{0} \rightarrow \Lambda_{b}^{0}}$ & 0.099 & $\stackrel{\Xi_{2, A}^{0} \rightarrow \Lambda_{b}^{0}}{g_{2, A}}$ & -0.043 \\
\hline$f_{3, S}^{\Xi_{b b}^{0} \rightarrow \Lambda_{b}^{0}}$ & 0.008 & $g_{3, S}^{\Xi_{b b}^{0} \rightarrow \Lambda_{b}^{0}}$ & -0.298 & $f_{3, A}^{\Xi_{b b}^{0} \rightarrow \Lambda_{b}^{0}}$ & -0.057 & $g_{3, A}^{\Xi_{b b}^{0} \rightarrow \Lambda_{b}^{0}}$ & 0.191 \\
\hline$f_{1, S}^{\Xi_{b b}^{0,-} \rightarrow \Sigma_{b}^{0,-}}$ & 0.102 & $g_{1, S}^{\Xi_{b b}^{0,-} \rightarrow \Sigma_{b}^{0,-}}$ & 0.094 & $f_{1, A}^{\Xi_{b b}^{0,-} \rightarrow \Sigma_{b}^{0,-}}$ & 0.100 & $g_{1, A}^{\Xi_{b b}^{0,-} \rightarrow \Sigma_{b}^{0,-}}$ & -0.021 \\
\hline$f_{2, S}^{\Xi_{b b}^{0,-} \rightarrow \Sigma_{b}^{0,-}}$ & -0.150 & $g_{2, S}^{\Xi_{b b}^{0,-} \rightarrow \Sigma_{b}^{0,-}}$ & 0.012 & $f_{2, A}^{\Xi_{b b}^{0,-} \rightarrow \Sigma_{b}^{0,-}}$ & 0.104 & $g_{2, A}^{\Xi_{b b}^{0,-} \rightarrow \Sigma_{b}^{0,-}}$ & -0.037 \\
\hline$f_{3, S}^{\Xi_{b b}^{0,-} \rightarrow \Sigma_{b}^{0,-}}$ & -0.004 & $g_{3, S}^{\Xi_{b b}^{0,-}} \rightarrow \Sigma_{b}^{0,-}$ & -0.222 & $f_{3, A}^{\Xi_{b b}^{0,-} \rightarrow \Sigma_{b}^{0,-}}$ & -0.070 & $g_{3, A}^{\Xi_{b b}^{0,-}} \rightarrow \Sigma_{b}^{0,-}$ & 0.183 \\
\hline$f_{1, S}^{\Omega_{b b}^{-} \rightarrow \Xi_{b}^{-}}$ & 0.098 & $g_{1, S}^{\Omega_{b b}^{-} \rightarrow \Xi_{b}^{-}}$ & 0.086 & $f_{1, A}^{\Omega_{b b}^{-} \rightarrow \Xi_{b}^{-}}$ & 0.095 & $g_{1, A}^{\Omega_{b b}^{-} \rightarrow \Xi_{b}^{-}}$ & -0.020 \\
\hline$f_{2, S}^{\Omega_{b b}^{-} \rightarrow \Xi_{b}^{-}}$ & -0.137 & $g_{2, S}^{\Omega_{b b}^{-}} \rightarrow \Xi_{b}^{-}$ & 0.034 & $f_{2, A}^{\Omega_{b b}^{-} \rightarrow \Xi_{b}^{-}}$ & 0.098 & $g_{2, A}^{\Omega_{b b}^{-} \rightarrow \Xi_{b}^{-}}$ & -0.040 \\
\hline$f_{3, S}^{\Omega_{b b}^{-} \rightarrow \Xi_{b}^{-}}$ & 0.004 & $g_{3, S}^{\Omega_{b b}^{-} \rightarrow \Xi_{b}^{-}}$ & -0.282 & $f_{3, A}^{\Omega_{b b}^{-} \rightarrow \Xi_{b}^{-}}$ & -0.059 & $\begin{array}{l}\Omega_{b b}^{-} \rightarrow \Xi_{b}^{-} \\
g_{3, A}\end{array}$ & 0.187 \\
\hline$f_{1, S}^{\Omega_{b b}^{-} \rightarrow \Xi_{b}^{\prime-}}$ & 0.099 & $g_{1, S}^{\Omega_{b b}^{-} \rightarrow \Xi_{b}^{\prime-}}$ & 0.091 & $f_{1, A}^{\Omega_{b b}^{-} \rightarrow \Xi_{b}^{\prime-}}$ & 0.097 & $g_{1, A}^{\Omega_{b b}^{-} \rightarrow \Xi_{b}^{\prime-}}$ & -0.021 \\
\hline$f_{2, S}^{\Omega_{b b}^{-} \rightarrow \Xi_{b}^{\prime-}}$ & -0.147 & $g_{2, S}^{\Omega_{b b}^{-}} \rightarrow \Xi_{b}^{\prime-}$ & 0.013 & $f_{2, A}^{\Omega_{b b}^{-} \rightarrow \Xi_{b}^{\prime-}}$ & 0.102 & $\begin{array}{l}\Omega_{b b}^{-} \rightarrow \Xi_{b}^{\prime-} \\
g_{2, A}\end{array}$ & -0.036 \\
\hline$f_{3, S}^{\Omega_{b b}^{-} \rightarrow \Xi_{b}^{\prime-}}$ & -0.005 & $g_{3, S}^{\Omega_{b b}^{-}} \rightarrow \Xi_{b}^{\prime-}$ & -0.226 & $f_{3, A}^{\Omega_{b b}^{-} \rightarrow \Xi_{b}^{\prime-}}$ & -0.068 & $\begin{array}{l}g_{3, A}^{-} \rightarrow \Xi_{b}^{\prime-} \\
\end{array}$ & 0.181 \\
\hline
\end{tabular}


TABLE X. Same as Table VI for $b \rightarrow d$ process.

\begin{tabular}{|c|c|c|c|c|c|c|c|}
\hline$F$ & $F(0)$ & $F$ & $F(0)$ & $F$ & $F(0)$ & $F$ & $F(0)$ \\
\hline$f_{2, S}^{T, \Xi_{b b}^{0} \rightarrow \Lambda_{b}^{0}}$ & 0.075 & $g_{2, S}^{T, \Xi_{b b}^{0} \rightarrow \Lambda_{b}^{0}}$ & 0.091 & $f_{2, A}^{T, \Xi_{b b}^{0} \rightarrow \Lambda_{b}^{0}}$ & -0.049 & $g_{2, A}^{T, \Xi_{b b}^{0} \rightarrow \Lambda_{b}^{0}}$ & -0.035 \\
\hline$f_{3, S}^{T, \Xi_{b b}^{0} \rightarrow \Lambda_{b}^{0}}$ & 0.072 & $g_{3, S}^{T, \Xi_{b b}^{0} \rightarrow \Lambda_{b}^{0}}$ & 0.114 & $f_{3, A}^{T, \Xi_{b b}^{0} \rightarrow \Lambda_{b}^{0}}$ & 0.104 & $g_{3, A}^{T, \Xi_{b b}^{0} \rightarrow \Lambda_{b}^{0}}$ & 0.028 \\
\hline$f_{2, S}^{T, \Xi_{b b}^{0,-} \rightarrow \Sigma_{b}^{0,-}}$ & 0.083 & $g_{2, S}^{T, \Xi_{b b}^{0,-} \rightarrow \Sigma_{b}^{0,-}}$ & 0.090 & $f_{2, A}^{T, \Xi_{b b}^{0,-} \rightarrow \Sigma_{b}^{0,-}}$ & -0.051 & $g_{2, A}^{T, \Xi_{b b}^{0,-} \rightarrow \Sigma_{b}^{0,-}}$ & -0.035 \\
\hline$f_{3, S}^{T, \Xi_{b b}^{0,-} \rightarrow \Sigma_{b}^{0,-}}$ & 0.072 & $g_{3, S}^{T, \Xi_{b b}^{0,-} \rightarrow \Sigma_{b}^{0,-}}$ & 0.154 & $f_{3, A}^{T, \Xi_{b b}^{0,-} \rightarrow \Sigma_{b}^{0,-}}$ & 0.104 & $g_{3, A}^{T, \Xi_{b b}^{0,-} \rightarrow \Sigma_{b}^{0,-}}$ & 0.023 \\
\hline$f_{2, S}^{T, \Omega_{b b}^{-}} \rightarrow \Xi_{b}^{-}$ & 0.074 & $g_{2, S}^{T, \Omega_{b b}^{-}} \rightarrow \Xi_{b}^{-}$ & 0.088 & $f_{2, A}^{T, \Omega_{b b}^{-} \rightarrow \Xi_{b}^{-}}$ & -0.048 & $g_{2, A}^{T, \Omega_{b b}^{-}} \rightarrow \Xi_{b}^{-}$ & -0.034 \\
\hline$f_{3, S}^{T, \Omega_{b b}^{-}} \rightarrow \Xi_{b}^{-}$ & 0.069 & $g_{3, S}^{T, \Omega_{b b}^{-}} \rightarrow \Xi_{b}^{-}$ & 0.119 & $f_{3, A}^{T, \Omega_{b b}^{-}} \rightarrow \Xi_{b}^{-}$ & 0.100 & $g_{3, A}^{T, \Omega_{b b}^{-}} \rightarrow \Xi_{b}^{-}$ & 0.026 \\
\hline$f_{2, S}^{T, \Omega_{b b}^{-} \rightarrow \Xi_{b}^{\prime-}}$ & 0.080 & $g_{2, S}^{T, \Omega_{b b}^{-}} \rightarrow \Xi_{b}^{\prime-}$ & 0.087 & $f_{2, A}^{T, \Omega_{b b}^{-} \rightarrow \Xi_{b}^{\prime-}}$ & -0.049 & $g_{2, A}^{T, \Omega_{b b}^{-}} \rightarrow \Xi_{b}^{\prime-}$ & -0.034 \\
\hline$f_{3, S}^{T, \Omega_{b b}^{-} \rightarrow \Xi_{b}^{\prime-}}$ & 0.069 & $g_{3, S}^{T, \Omega_{b b}^{-} \rightarrow \Xi_{b}^{\prime-}}$ & 0.148 & $f_{3, A}^{T, \Omega_{b b}^{-} \rightarrow \Xi_{b}^{\prime-}}$ & 0.101 & $g_{3, A}^{T, \Omega_{b b}^{-} \rightarrow \Xi_{b}^{\prime-}}$ & 0.023 \\
\hline
\end{tabular}

TABLE XI. Same as Table VII but for $b \rightarrow d$ process.

\begin{tabular}{|c|c|c|c|c|c|c|c|}
\hline$F$ & $F(0)$ & $F$ & $F(0)$ & $F$ & $F(0)$ & $F$ & $F(0)$ \\
\hline$f_{1, S}^{\Xi_{b c}^{+} \rightarrow \Lambda_{c}^{+}}$ & 0.143 & $g_{1, S}^{\Xi_{b c}^{+} \rightarrow \Lambda_{c}^{+}}$ & 0.117 & $f_{1, A}^{\Xi_{b c}^{+} \rightarrow \Lambda_{c}^{+}}$ & 0.130 & $g_{1, A}^{\Xi_{b c}^{+} \rightarrow \Lambda_{c}^{+}}$ & -0.020 \\
\hline$f_{2, S}^{\Xi_{b c}^{+}} \rightarrow \Lambda_{c}^{+}$ & -0.055 & $g_{2, S}^{\Xi_{b c}^{+}} \rightarrow \Lambda_{c}^{+}$ & 0.070 & $f_{2, A}^{\Xi_{b c}^{+} \rightarrow \Lambda_{c}^{+}}$ & 0.149 & $\frac{\Xi_{2, A}^{+}}{g_{b c}} \rightarrow \Lambda_{c}^{+}$ & -0.054 \\
\hline$f_{3, S}^{\Xi_{b c}^{+} \rightarrow \Lambda_{c}^{+}}$ & 0.009 & $g_{3, S}^{\Xi_{b c}^{+} \rightarrow \Lambda_{c}^{+}}$ & -0.224 & $f_{3, A}^{\Xi_{b c}^{+} \rightarrow \Lambda_{c}^{+}}$ & -0.087 & $g_{3, A}^{\Xi_{b c}^{+} \rightarrow \Lambda_{c}^{+}}$ & 0.121 \\
\hline$f_{1, S}^{\Xi_{b c}^{+, 0} \rightarrow \Sigma_{c}^{+, 0}}$ & 0.143 & $g_{1, S}^{\Xi_{b c}^{+, 0} \rightarrow \Sigma_{c}^{+, 0}}$ & 0.123 & $f_{1, A}^{\Xi_{b c}^{+, 0} \rightarrow \Sigma_{c}^{+, 0}}$ & 0.130 & $g_{1, A}^{\Xi_{b c}^{+, 0}} \rightarrow \Sigma_{c}^{+, 0}$ & -0.021 \\
\hline$f_{2, S}^{\Xi_{b c}^{+, 0} \rightarrow \Sigma_{c}^{+, 0}}$ & -0.067 & $g_{2, S}^{\Xi_{b c}^{+, 0}} \rightarrow \Sigma_{c}^{+, 0}$ & 0.046 & $f_{2, A}^{\Xi_{b c}^{+, 0} \rightarrow \Sigma_{c}^{+, 0}}$ & 0.150 & $g_{2, A}^{\Xi_{b c}^{+, 0}} \rightarrow \Sigma_{c}^{+, 0}$ & -0.050 \\
\hline$f_{3, S}^{\Xi_{b c}^{+, 0} \rightarrow \Sigma_{c}^{+, 0}}$ & 0.001 & $g_{3, S}^{\Xi_{b c}^{+, 0}} \rightarrow \Sigma_{c}^{+, 0}$ & -0.197 & $f_{3, A}^{\Xi_{b c}^{+, 0} \rightarrow \Sigma_{c}^{+, 0}}$ & -0.094 & $g_{3, A}^{\Xi_{b c}^{+, 0}} \rightarrow \Sigma_{c}^{+, 0}$ & 0.121 \\
\hline$f_{1, S}^{\Omega_{b c}^{0} \rightarrow \Xi_{c}^{0}}$ & 0.133 & $g_{1, S}^{\Omega_{b c}^{0} \rightarrow \Xi_{c}^{0}}$ & 0.111 & $f_{1, A}^{\Omega_{b c}^{0} \rightarrow \Xi_{c}^{0}}$ & 0.122 & $g_{1, A}^{\Omega_{b c}^{0} \rightarrow \Xi_{c}^{0}}$ & -0.019 \\
\hline$f_{2, S}^{\Omega_{b c}^{0} \rightarrow \Xi_{c}^{0}}$ & -0.060 & $g_{2, S}^{\Omega_{b c}^{0} \rightarrow \Xi_{c}^{0}}$ & 0.053 & $f_{2, A}^{\Omega_{b b}^{0} \rightarrow \Xi_{c}^{0}}$ & 0.139 & $g_{2, A}^{\Omega_{b c}^{0} \rightarrow \Xi_{c}^{0}}$ & -0.049 \\
\hline$f_{3, S}^{\Omega_{b c}^{0} \rightarrow \Xi_{c}^{0}}$ & 0.003 & $g_{3, S}^{\Omega_{b c}^{0} \rightarrow \Xi_{c}^{0}}$ & -0.204 & $f_{3, A}^{\Omega_{b c}^{0} \rightarrow \Xi_{c}^{0}}$ & -0.085 & $\begin{array}{l}g_{b, A}^{0} \rightarrow \Xi_{c}^{0} \\
g_{3, A}\end{array}$ & 0.118 \\
\hline$f_{1, S}^{\Omega_{b c}^{0} \rightarrow \Xi_{c}^{\prime 0}}$ & 0.133 & $g_{1, S}^{\Omega_{b c}^{0} \rightarrow \Xi_{c}^{\prime 0}}$ & 0.116 & $f_{1, A}^{\Omega_{b c}^{0} \rightarrow \Xi_{c}^{\prime 0}}$ & 0.122 & $g_{1, A}^{\Omega_{b c}^{0} \rightarrow \Xi_{c}^{\prime 0}}$ & -0.020 \\
\hline$f_{2, S}^{\Omega_{b c}^{0} \rightarrow \Xi_{c}^{\prime 0}}$ & -0.067 & $g_{2, S}^{\Omega_{b c}^{0} \rightarrow \Xi_{c}^{\prime 0}}$ & 0.038 & $f_{2, A}^{\Omega_{b c}^{0} \rightarrow \Xi_{c}^{\prime 0}}$ & 0.140 & $g_{2, A}^{\Omega_{b c}^{0} \rightarrow \Xi_{c}^{\prime 0}}$ & -0.047 \\
\hline$f_{3, S}^{\Omega_{b c}^{0} \rightarrow \Xi_{c}^{\prime 0}}$ & -0.001 & $\begin{array}{l}g_{3, S}^{0} \rightarrow \Xi_{c}^{\prime 0} \\
g^{\prime}\end{array}$ & -0.185 & $f_{3, A}^{\Omega_{b c}^{0} \rightarrow \Xi_{c}^{\prime 0}}$ & -0.089 & $g_{3, A}^{\Omega_{b c}^{0}} \rightarrow \Xi_{c}^{\prime 0}$ & 0.118 \\
\hline
\end{tabular}

TABLE XII. Same as Table VIII but for $b \rightarrow d$ process.

\begin{tabular}{|c|c|c|c|c|c|c|c|}
\hline$F$ & $F(0)$ & $F$ & $F(0)$ & $F$ & $F(0)$ & $F$ & $F(0)$ \\
\hline$f_{2, S}^{T, \Xi_{b c}^{+} \rightarrow \Lambda_{c}^{+}}$ & 0.110 & $g_{2, S}^{T, \Xi_{b c}^{+}} \rightarrow \Lambda_{c}^{+}$ & 0.142 & $f_{2, A}^{T, \Xi_{b c}^{+}} \rightarrow \Lambda_{c}^{+}$ & -0.052 & $g_{2, A}^{T, \Xi_{b c}^{+} \rightarrow \Lambda_{c}^{+}}$ & -0.052 \\
\hline$f_{3, S}^{T, \Xi_{b c}^{+}} \rightarrow \Lambda_{c}^{+}$ & 0.068 & $g_{3, S}^{T, \Xi_{b c}^{+}} \rightarrow \Lambda_{c}^{+}$ & -0.010 & $f_{3, A}^{T, \Xi_{b c}^{+}} \rightarrow \Lambda_{c}^{+}$ & 0.133 & $g_{3, A}^{T, \Xi_{b c}^{+} \rightarrow \Lambda_{c}^{+}}$ & 0.055 \\
\hline$f_{2, S}^{T, \Xi_{b c}^{+, 0} \rightarrow \Sigma_{c}^{+, 0}}$ & 0.119 & $g_{2, S}^{T, \Xi_{b c}^{+, 0} \rightarrow \Sigma_{c}^{+, 0}}$ & 0.140 & $f_{2, A}^{T, \Xi_{b c}^{+, 0} \rightarrow \Sigma_{c}^{+, 0}}$ & -0.053 & $g_{2, A}^{T, \Xi_{b c}^{+, 0} \rightarrow \Sigma_{c}^{+, 0}}$ & -0.052 \\
\hline$f_{3, S}^{T, \Xi_{b c}^{+, 0}} \rightarrow \Sigma_{c}^{+, 0}$ & 0.064 & $g_{3, S}^{T, \Xi_{b c}^{+, 0} \rightarrow \Sigma_{c}^{+, 0}}$ & 0.006 & $f_{3, A}^{T, \Xi_{b c}^{+, 0} \rightarrow \Sigma_{c}^{+, 0}}$ & 0.130 & $\begin{array}{l}T, \Xi_{b c}^{+, 0} \rightarrow \Sigma_{c}^{+, 0} \\
g_{3, A}\end{array}$ & 0.055 \\
\hline$f_{2, S}^{T, \Omega_{b c}^{0} \rightarrow \Xi_{c}^{0}}$ & 0.105 & $g_{2, S}^{T, \Omega_{b c}^{0}} \rightarrow \Xi_{c}^{0}$ & 0.131 & $f_{2, A}^{T, \Omega_{b c}^{0} \rightarrow \Xi_{c}^{0}}$ & -0.050 & $g_{2, A}^{T, \Omega_{b c}^{0} \rightarrow \Xi_{c}^{0}}$ & -0.049 \\
\hline$f_{3, S}^{T, \Omega_{b c}^{0} \rightarrow \Xi_{c}^{0}}$ & 0.064 & $g_{3, S}^{T, \Omega_{b c}^{0}} \rightarrow \Xi_{c}^{0}$ & -0.001 & $f_{3, A}^{T, \Omega_{b c}^{0} \rightarrow \Xi_{c}^{0}}$ & 0.124 & $g_{3, A}^{T, \Omega_{b c}^{0} \rightarrow \Xi_{c}^{0}}$ & 0.053 \\
\hline$f_{2, S}^{T, \Omega_{b c}^{0} \rightarrow \Xi_{c}^{\prime 0}}$ & 0.110 & $g_{2, S}^{T, \Omega_{b c}^{0} \rightarrow \Xi_{c}^{\prime 0}}$ & 0.129 & $f_{2, A}^{T, \Omega_{b c}^{0} \rightarrow \Xi_{c}^{\prime 0}}$ & -0.051 & $g_{2, A}^{T, \Omega_{b c}^{0}} \rightarrow \Xi_{c}^{\prime 0}$ & -0.049 \\
\hline$f_{3, S}^{T, \Omega_{b c}^{0} \rightarrow \Xi_{c}^{\prime 0}}$ & 0.062 & $g_{3, S}^{T, \Omega_{b c}^{0} \rightarrow \Xi_{c}^{\prime 0}}$ & 0.010 & $f_{3, A}^{T, \Omega_{b c}^{0} \rightarrow \Xi_{c}^{\prime 0}}$ & 0.123 & $g_{3, A}^{T, \Omega_{b c}^{0} \rightarrow \Xi_{c}^{\prime 0}}$ & 0.053 \\
\hline
\end{tabular}


TABLE XIII. Decay widths and branching ratios for $b \rightarrow s$ process in $b b$ sector.

\begin{tabular}{lccc}
\hline \hline Channels & $\Gamma / \mathrm{GeV}$ & $\mathcal{B}$ & $\Gamma_{L} / \Gamma_{T}$ \\
\hline$\Xi_{b b}^{0} \rightarrow \Xi_{b}^{0} e^{+} e^{-}$ & $1.98 \times 10^{-19}$ & $1.11 \times 10^{-7}$ & 3.48 \\
$\Xi_{b b}^{0} \rightarrow \Xi_{b}^{\prime 0} e^{+} e^{-}$ & $5.20 \times 10^{-19}$ & $2.92 \times 10^{-7}$ & 0.70 \\
$\Xi_{b b}^{-} \rightarrow \Xi_{b}^{-} e^{+} e^{-}$ & $1.97 \times 10^{-19}$ & $1.11 \times 10^{-7}$ & 3.49 \\
$\Xi_{b b}^{-} \rightarrow \Xi_{b}^{\prime-} e^{+} e^{-}$ & $5.20 \times 10^{-19}$ & $2.92 \times 10^{-7}$ & 0.70 \\
$\Omega_{b b}^{-} \rightarrow \Omega_{b}^{-} e^{+} e^{-}$ & $1.02 \times 10^{-18}$ & $1.25 \times 10^{-6}$ & 0.70 \\
$\Xi_{b b}^{0} \rightarrow \Xi_{b}^{0} \mu^{+} \mu^{-}$ & $1.92 \times 10^{-19}$ & $1.08 \times 10^{-7}$ & 3.95 \\
$\Xi_{b b}^{0} \rightarrow \Xi_{b}^{\prime 0} \mu^{+} \mu^{-}$ & $4.47 \times 10^{-19}$ & $2.52 \times 10^{-7}$ & 0.91 \\
$\Xi_{b b}^{-} \rightarrow \Xi_{b}^{-} \mu^{+} \mu^{-}$ & $1.91 \times 10^{-19}$ & $1.08 \times 10^{-7}$ & 3.96 \\
$\Xi_{b b}^{-} \rightarrow \Xi_{b}^{\prime-} \mu^{+} \mu^{-}$ & $4.47 \times 10^{-19}$ & $2.52 \times 10^{-7}$ & 0.91 \\
$\Omega_{b b}^{-} \rightarrow \Omega_{b}^{-} \mu^{+} \mu^{-}$ & $8.85 \times 10^{-19}$ & $1.08 \times 10^{-6}$ & 0.90 \\
$\Xi_{b b}^{0} \rightarrow \Xi_{b}^{0} \tau^{+} \tau^{-}$ & $3.72 \times 10^{-20}$ & $2.09 \times 10^{-8}$ & 6.17 \\
$\Xi_{b b}^{0} \rightarrow \Xi_{b}^{\prime 0} \tau^{+} \tau^{-}$ & $4.87 \times 10^{-20}$ & $2.74 \times 10^{-8}$ & 1.02 \\
$\Xi_{b b}^{-} \rightarrow \Xi_{b}^{-} \tau^{+} \tau^{-}$ & $3.69 \times 10^{-20}$ & $2.07 \times 10^{-8}$ & 6.18 \\
$\Xi_{b b}^{-} \rightarrow \Xi_{b}^{\prime-} \tau^{+} \tau^{-}$ & $4.87 \times 10^{-20}$ & $2.74 \times 10^{-8}$ & 1.02 \\
$\Omega_{b b}^{-} \rightarrow \Omega_{b}^{-} \tau^{+} \tau^{-}$ & $1.02 \times 10^{-19}$ & $1.24 \times 10^{-7}$ & 1.00 \\
\hline \hline
\end{tabular}

\section{Results for phenomenological observables}

The decay widths are shown in Tables XIII-XV for $b \rightarrow s$ process and Tables XVI-XVIII for $b \rightarrow d$ process. Some comments are given in order.

(i) Since there exist uncertainties in the lifetimes of the parent baryons, there may exist small fluctuations in the results for branching ratios.

(ii) It can be seen from these tables that, the decay widths are very close to each other for $l=e / \mu$ cases, while it is roughly one order of magnitude smaller for $l=\tau$ case. This can be attributed to the much smaller phase space for $l=\tau$ case.

TABLE XIV. Decay widths and branching ratios for $b \rightarrow s$ process in $b c$ sector.

\begin{tabular}{lccc}
\hline \hline Channels & $\Gamma / \mathrm{GeV}$ & $\mathcal{B}$ & $\Gamma_{L} / \Gamma_{T}$ \\
\hline$\Xi_{b c}^{+} \rightarrow \Xi_{c}^{+} e^{+} e^{-}$ & $1.46 \times 10^{-19}$ & $5.43 \times 10^{-8}$ & 2.92 \\
$\Xi_{b c}^{+} \rightarrow \Xi_{c}^{\prime+} e^{+} e^{-}$ & $4.54 \times 10^{-19}$ & $1.69 \times 10^{-7}$ & 0.68 \\
$\Xi_{b c}^{0} \rightarrow \Xi_{c}^{0} e^{+} e^{-}$ & $1.46 \times 10^{-19}$ & $2.06 \times 10^{-8}$ & 2.93 \\
$\Xi_{b c}^{0} \rightarrow \Xi_{c}^{\prime 0} e^{+} e^{-}$ & $4.53 \times 10^{-19}$ & $6.40 \times 10^{-8}$ & 0.68 \\
$\Omega_{b c}^{0} \rightarrow \Omega_{c}^{0} e^{+} e^{-}$ & $7.42 \times 10^{-19}$ & $2.48 \times 10^{-7}$ & 0.68 \\
$\Xi_{b c}^{+} \rightarrow \Xi_{c}^{+} \mu^{+} \mu^{-}$ & $1.40 \times 10^{-19}$ & $5.21 \times 10^{-8}$ & 3.44 \\
$\Xi_{b c}^{+} \rightarrow \Xi_{c}^{\prime+} \mu^{+} \mu^{-}$ & $3.97 \times 10^{-19}$ & $1.47 \times 10^{-7}$ & 0.86 \\
$\Xi_{b c}^{0} \rightarrow \Xi_{c}^{0} \mu^{+} \mu^{-}$ & $1.40 \times 10^{-19}$ & $1.98 \times 10^{-8}$ & 3.45 \\
$\Xi_{b c}^{0} \rightarrow \Xi_{c}^{\prime} \mu^{+} \mu^{-}$ & $3.95 \times 10^{-19}$ & $5.59 \times 10^{-8}$ & 0.86 \\
$\Omega_{b c}^{0} \rightarrow \Omega_{c}^{0} \mu^{+} \mu^{-}$ & $6.41 \times 10^{-19}$ & $2.14 \times 10^{-7}$ & 0.88 \\
$\Xi_{b c}^{+} \rightarrow \Xi_{c}^{+} \tau^{+} \tau^{-}$ & $3.02 \times 10^{-20}$ & $1.12 \times 10^{-8}$ & 4.19 \\
$\Xi_{b c}^{+} \rightarrow \Xi_{c}^{\prime+} \tau^{+} \tau^{-}$ & $6.50 \times 10^{-20}$ & $2.41 \times 10^{-8}$ & 0.99 \\
$\Xi_{b c}^{0} \rightarrow \Xi_{c}^{0} \tau^{+} \tau^{-}$ & $2.98 \times 10^{-20}$ & $4.22 \times 10^{-9}$ & 4.20 \\
$\Xi_{b c}^{0} \rightarrow \Xi_{c}^{\prime 0} \tau^{+} \tau^{-}$ & $6.45 \times 10^{-20}$ & $9.12 \times 10^{-9}$ & 0.99 \\
$\Omega_{b c}^{0} \rightarrow \Omega_{c}^{0} \tau^{+} \tau^{-}$ & $9.12 \times 10^{-20}$ & $3.05 \times 10^{-8}$ & 0.99 \\
\hline \hline
\end{tabular}

TABLE XV. Decay widths and branching ratios for $b \rightarrow s$ process in $b c^{\prime}$ sector.

\begin{tabular}{lccc}
\hline \hline Channels & $\Gamma / \mathrm{GeV}$ & $\mathcal{B}$ & $\Gamma_{L} / \Gamma_{T}$ \\
\hline$\Xi_{b c}^{\prime+} \rightarrow \Xi_{c}^{+} e^{+} e^{-}$ & $1.93 \times 10^{-19}$ & $7.16 \times 10^{-8}$ & 0.58 \\
$\Xi_{b c}^{\prime+} \rightarrow \Xi_{c}^{\prime+} e^{+} e^{-}$ & $1.27 \times 10^{-19}$ & $4.70 \times 10^{-8}$ & 3.16 \\
$\Xi_{b c}^{\prime 0} \rightarrow \Xi_{c}^{0} e^{+} e^{-}$ & $1.92 \times 10^{-19}$ & $2.72 \times 10^{-8}$ & 0.58 \\
$\Xi_{b c}^{\prime 0} \rightarrow \Xi_{c}^{\prime 0} e^{+} e^{-}$ & $1.26 \times 10^{-19}$ & $1.79 \times 10^{-8}$ & 3.16 \\
$\Omega_{b c}^{\prime} \rightarrow \Omega_{c}^{0} e^{+} e^{-}$ & $2.11 \times 10^{-19}$ & $7.05 \times 10^{-8}$ & 3.34 \\
$\Xi_{b c}^{\prime+} \rightarrow \Xi_{c}^{+} \mu^{+} \mu^{-}$ & $1.69 \times 10^{-19}$ & $6.27 \times 10^{-8}$ & 0.71 \\
$\Xi_{b c}^{\prime+} \rightarrow \Xi_{c}^{\prime+} \mu^{+} \mu^{-}$ & $1.21 \times 10^{-19}$ & $4.48 \times 10^{-8}$ & 3.87 \\
$\Xi_{b c}^{\prime 0} \rightarrow \Xi_{c}^{0} \mu^{+} \mu^{-}$ & $1.68 \times 10^{-19}$ & $2.38 \times 10^{-8}$ & 0.71 \\
$\Xi_{b c}^{\prime 0} \rightarrow \Xi_{c}^{\prime} \mu^{+} \mu^{-}$ & $1.20 \times 10^{-19}$ & $1.70 \times 10^{-8}$ & 3.88 \\
$\Omega_{b c}^{\prime 0} \rightarrow \Omega_{c}^{0} \mu^{+} \mu^{-}$ & $2.01 \times 10^{-19}$ & $6.71 \times 10^{-8}$ & 4.15 \\
$\Xi_{b c}^{\prime+} \rightarrow \Xi_{c}^{+} \tau^{+} \tau^{-}$ & $3.27 \times 10^{-20}$ & $1.21 \times 10^{-8}$ & 0.71 \\
$\Xi_{b c}^{\prime+} \rightarrow \Xi_{c}^{\prime+} \tau^{+} \tau^{-}$ & $2.03 \times 10^{-20}$ & $7.53 \times 10^{-9}$ & 4.56 \\
$\Xi_{b c}^{\prime 0} \rightarrow \Xi_{c}^{0} \tau^{+} \tau^{-}$ & $3.23 \times 10^{-20}$ & $4.57 \times 10^{-9}$ & 0.71 \\
$\Xi_{b c}^{\prime 0} \rightarrow \Xi_{c}^{\prime 0} \tau^{+} \tau^{-}$ & $2.01 \times 10^{-20}$ & $2.85 \times 10^{-9}$ & 4.56 \\
$\Omega_{b c}^{\prime 0} \rightarrow \Omega_{c}^{0} \tau^{+} \tau^{-}$ & $2.91 \times 10^{-20}$ & $9.74 \times 10^{-9}$ & 4.85 \\
\hline \hline
\end{tabular}

(iii) Most of the branching ratios are $10^{-8}-10^{-7}$ for $b \rightarrow s$ process and $10^{-9}-10^{-8}$ for $b \rightarrow d$ process, which are roughly one order of magnitude smaller than the corresponding mesonic cases. This is because we believe that the lifetime of the doubly heavy baryon is roughly one order of magnitude smaller than that of $B$ meson.

The differential decay widths for $\Xi_{b b}^{0} \rightarrow \Xi_{b}^{0} l^{+} l^{-}$with $l=e, \mu, \tau$ are plotted in Fig. 3, where the resonant contributions are not taken into account. It can be seen that the curves for $l=e / \mu$ almost coincide with each other and the much smaller phase space for $l=\tau$ case can be seen clearly. The curves of forward-backward asymmetry

TABLE XVI. Decay widths and branching ratios for $b \rightarrow d$ process in $b b$ sector.

\begin{tabular}{lccc}
\hline \hline Channels & $\Gamma / \mathrm{GeV}$ & $\mathcal{B}$ & $\Gamma_{L} / \Gamma_{T}$ \\
\hline$\Xi_{b b}^{0} \rightarrow \Lambda_{b}^{0} e^{+} e^{-}$ & $6.46 \times 10^{-21}$ & $3.63 \times 10^{-9}$ & 3.22 \\
$\Xi_{b b}^{0} \rightarrow \Sigma_{b}^{0} e^{+} e^{-}$ & $1.60 \times 10^{-20}$ & $9.00 \times 10^{-9}$ & 0.70 \\
$\Xi_{b b}^{-} \rightarrow \Sigma_{b}^{-} e^{+} e^{-}$ & $3.19 \times 10^{-20}$ & $1.79 \times 10^{-8}$ & 0.70 \\
$\Omega_{b b}^{-} \rightarrow \Xi_{b}^{-} e^{+} e^{-}$ & $5.71 \times 10^{-21}$ & $6.94 \times 10^{-9}$ & 3.36 \\
$\Omega_{b b}^{-} \rightarrow \Xi_{b}^{\prime} e^{+} e^{-}$ & $1.54 \times 10^{-20}$ & $1.88 \times 10^{-8}$ & 0.70 \\
$\Xi_{b b}^{0} \rightarrow \Lambda_{b}^{0} \mu^{+} \mu^{-}$ & $6.32 \times 10^{-21}$ & $3.55 \times 10^{-9}$ & 3.51 \\
$\Xi_{b b}^{0} \rightarrow \Sigma_{b}^{0} \mu^{+} \mu^{-}$ & $1.41 \times 10^{-20}$ & $7.94 \times 10^{-9}$ & 0.88 \\
$\Xi_{b b}^{-} \rightarrow \Sigma_{b}^{-} \mu^{+} \mu^{-}$ & $2.81 \times 10^{-20}$ & $1.58 \times 10^{-8}$ & 0.88 \\
$\Omega_{b b}^{-} \rightarrow \Xi_{b}^{-} \mu^{+} \mu^{-}$ & $5.58 \times 10^{-21}$ & $6.78 \times 10^{-9}$ & 3.70 \\
$\Omega_{b b}^{-} \rightarrow \Xi_{b}^{\prime} \mu^{+} \mu^{-}$ & $1.36 \times 10^{-20}$ & $1.66 \times 10^{-8}$ & 0.87 \\
$\Xi_{b b}^{0} \rightarrow \Lambda_{b}^{0} \tau^{+} \tau^{-}$ & $1.75 \times 10^{-21}$ & $9.86 \times 10^{-10}$ & 5.59 \\
$\Xi_{b b}^{0} \rightarrow \Sigma_{b}^{0} \tau^{+} \tau^{-}$ & $2.10 \times 10^{-21}$ & $1.18 \times 10^{-9}$ & 1.01 \\
$\Xi_{b b}^{-} \rightarrow \Sigma_{b}^{-} \tau^{+} \tau^{-}$ & $4.17 \times 10^{-21}$ & $2.35 \times 10^{-9}$ & 1.01 \\
$\Omega_{b b}^{-} \rightarrow \Xi_{b}^{-} \tau^{+} \tau^{-}$ & $1.40 \times 10^{-21}$ & $1.71 \times 10^{-9}$ & 5.80 \\
$\Omega_{b b}^{-} \rightarrow \Xi_{b}^{\prime} \tau^{+} \tau^{-}$ & $2.08 \times 10^{-21}$ & $2.53 \times 10^{-9}$ & 1.01 \\
\hline \hline
\end{tabular}


TABLE XVII. Decay widths and branching ratios for $b \rightarrow d$ process in $b c$ sector.

\begin{tabular}{lccc}
\hline \hline Channels & $\Gamma / \mathrm{GeV}$ & $\mathcal{B}$ & $\Gamma_{L} / \Gamma_{T}$ \\
\hline$\Xi_{b c}^{+} \rightarrow \Lambda_{c}^{+} e^{+} e^{-}$ & $4.54 \times 10^{-21}$ & $1.68 \times 10^{-9}$ & 2.72 \\
$\Xi_{b c}^{+} \rightarrow \Sigma_{c}^{+} e^{+} e^{-}$ & $1.34 \times 10^{-20}$ & $4.97 \times 10^{-9}$ & 0.68 \\
$\Xi_{b c}^{0} \rightarrow \Sigma_{c}^{0} e^{+} e^{-}$ & $2.67 \times 10^{-20}$ & $3.78 \times 10^{-9}$ & 0.68 \\
$\Omega_{b c}^{0} \rightarrow \Xi_{c}^{0} e^{+} e^{-}$ & $3.28 \times 10^{-21}$ & $1.10 \times 10^{-9}$ & 3.10 \\
$\Omega_{b c}^{0} \rightarrow \Xi_{c}^{\prime 0} e^{+} e^{-}$ & $1.04 \times 10^{-20}$ & $3.47 \times 10^{-9}$ & 0.68 \\
$\Xi_{b c}^{+} \rightarrow \Lambda_{c}^{+} \mu^{+} \mu^{-}$ & $4.40 \times 10^{-21}$ & $1.63 \times 10^{-9}$ & 3.05 \\
$\Xi_{b c}^{+} \rightarrow \Sigma_{c}^{+} \mu^{+} \mu^{-}$ & $1.20 \times 10^{-20}$ & $4.44 \times 10^{-9}$ & 0.83 \\
$\Xi_{b c}^{0} \rightarrow \Sigma_{c}^{0} \mu^{+} \mu^{-}$ & $2.39 \times 10^{-20}$ & $3.38 \times 10^{-9}$ & 0.83 \\
$\Omega_{b c}^{0} \rightarrow \Xi_{c}^{0} \mu^{+} \mu^{-}$ & $3.16 \times 10^{-21}$ & $1.06 \times 10^{-9}$ & 3.58 \\
$\Omega_{b c}^{0} \rightarrow \Xi_{c}^{\prime 0} \mu^{+} \mu^{-}$ & $9.16 \times 10^{-21}$ & $3.06 \times 10^{-9}$ & 0.85 \\
$\Xi_{b c}^{+} \rightarrow \Lambda_{c}^{+} \tau^{+} \tau^{-}$ & $1.31 \times 10^{-21}$ & $4.87 \times 10^{-10}$ & 3.86 \\
$\Xi_{b c}^{+} \rightarrow \Sigma_{c}^{+} \tau^{+} \tau^{-}$ & $2.54 \times 10^{-21}$ & $9.43 \times 10^{-10}$ & 0.98 \\
$\Xi_{b c}^{0} \rightarrow \Sigma_{c}^{0} \tau^{+} \tau^{-}$ & $5.06 \times 10^{-21}$ & $7.16 \times 10^{-10}$ & 0.99 \\
$\Omega_{b c}^{0} \rightarrow \Xi_{c}^{0} \tau^{+} \tau^{-}$ & $7.46 \times 10^{-22}$ & $2.49 \times 10^{-10}$ & 4.38 \\
$\Omega_{b c}^{0} \rightarrow \Xi_{c}^{\prime 0} \tau^{+} \tau^{-}$ & $1.70 \times 10^{-21}$ & $5.70 \times 10^{-10}$ & 1.00 \\
\hline \hline
\end{tabular}

TABLE XVIII. Decay widths and branching ratios for $b \rightarrow d$ process in $b c^{\prime}$ sector.

\begin{tabular}{lccc}
\hline \hline Channels & $\Gamma / \mathrm{GeV}$ & $\mathcal{B}$ & $\Gamma_{L} / \Gamma_{T}$ \\
\hline$\Xi_{b c}^{\prime+} \rightarrow \Lambda_{c}^{+} e^{+} e^{-}$ & $6.61 \times 10^{-21}$ & $2.45 \times 10^{-9}$ & 0.54 \\
$\Xi_{b c}^{\prime+} \rightarrow \Sigma_{c}^{+} e^{+} e^{-}$ & $3.55 \times 10^{-21}$ & $1.32 \times 10^{-9}$ & 3.17 \\
$\Xi_{b c}^{\prime 0} \rightarrow \Sigma_{c}^{0} e^{+} e^{-}$ & $7.09 \times 10^{-21}$ & $1.00 \times 10^{-9}$ & 3.17 \\
$\Omega_{b c}^{\prime 0} \rightarrow \Xi_{c}^{0} e^{+} e^{-}$ & $4.59 \times 10^{-21}$ & $1.54 \times 10^{-9}$ & 0.55 \\
$\Omega_{b c}^{\prime 0} \rightarrow \Xi_{c}^{\prime 0} e^{+} e^{-}$ & $2.82 \times 10^{-21}$ & $9.43 \times 10^{-10}$ & 3.39 \\
$\Xi_{b c}^{\prime+} \rightarrow \Lambda_{c}^{+} \mu^{+} \mu^{-}$ & $5.98 \times 10^{-21}$ & $2.22 \times 10^{-9}$ & 0.63 \\
$\Xi_{b c}^{\prime+} \rightarrow \Sigma_{c}^{+} \mu^{+} \mu^{-}$ & $3.41 \times 10^{-21}$ & $1.26 \times 10^{-9}$ & 3.74 \\
$\Xi_{b c}^{\prime 0} \rightarrow \Sigma_{c}^{0} \mu^{+} \mu^{-}$ & $6.81 \times 10^{-21}$ & $9.62 \times 10^{-10}$ & 3.75 \\
$\Omega_{b c}^{\prime 0} \rightarrow \Xi_{c}^{0} \mu^{+} \mu^{-}$ & $4.06 \times 10^{-21}$ & $1.36 \times 10^{-9}$ & 0.67 \\
$\Omega_{b c}^{\prime 0} \rightarrow \Xi_{c}^{\prime 0} \mu^{+} \mu^{-}$ & $2.71 \times 10^{-21}$ & $9.05 \times 10^{-10}$ & 4.06 \\
$\Xi_{b c}^{\prime+} \rightarrow \Lambda_{c}^{+} \tau^{+} \tau^{-}$ & $1.60 \times 10^{-21}$ & $5.95 \times 10^{-10}$ & 0.65 \\
$\Xi_{b c}^{\prime+} \rightarrow \Sigma_{c}^{+} \tau^{+} \tau^{-}$ & $7.32 \times 10^{-22}$ & $2.71 \times 10^{-10}$ & 4.48 \\
$\Xi_{b c}^{\prime 0} \rightarrow \Sigma_{c}^{0} \tau^{+} \tau^{-}$ & $1.46 \times 10^{-21}$ & $2.06 \times 10^{-10}$ & 4.48 \\
$\Omega_{b c}^{\prime 0} \rightarrow \Xi_{c}^{0} \tau^{+} \tau^{-}$ & $8.80 \times 10^{-22}$ & $2.94 \times 10^{-10}$ & 0.68 \\
$\Omega_{b c}^{\prime 0} \rightarrow \Xi_{c}^{\prime 0} \tau^{+} \tau^{-}$ & $5.04 \times 10^{-22}$ & $1.69 \times 10^{-10}$ & 4.81 \\
\hline \hline
\end{tabular}

(FBA) for $\Xi_{b b}^{0} \rightarrow \Xi_{b}^{0} l^{+} l^{-}$with $l=e, \mu, \tau$ are plotted in Fig. 4. It can be seen from this figure that, the zero-crossing point is around $q^{2} \approx 2 \mathrm{GeV}^{2}$ for $l=e / \mu$ cases. The zerocrossing points for other $b \rightarrow s$ processes and for $b \rightarrow d$ processes can be found in Tables XIX and XX respectively. It can be seen from these tables that these $s_{0}$ roughly range from 2 to $3 \mathrm{GeV}^{2}$.

Following Ref. [52], we now analyse the zero-crossing point $s_{0}$ of FBA which satisfies

$$
\frac{d \bar{A}_{F B}}{d q^{2}}=\frac{I_{1}}{2\left(I_{0}-I_{2} / 3\right)}=0
$$

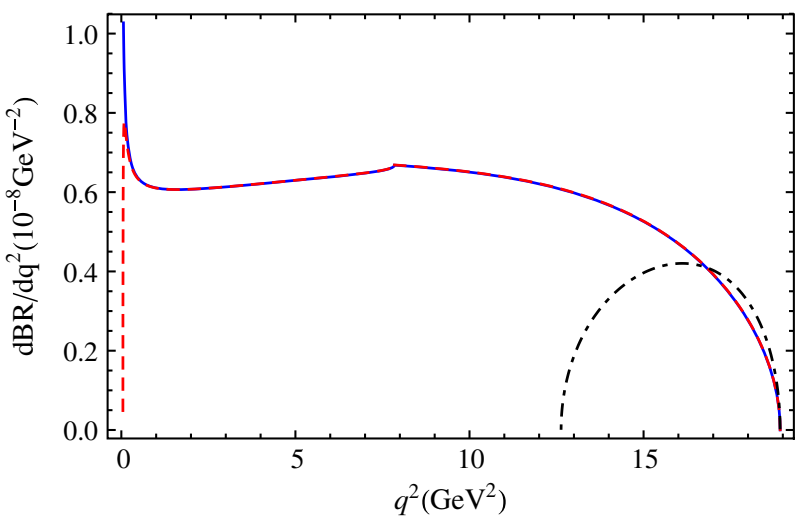

FIG. 3. $d \mathcal{B} / d q^{2}$ for $\Xi_{b b}^{0} \rightarrow \Xi_{b}^{0} l^{+} l^{-}$with $l=e, \mu, \tau$. The blue solid line, the red dashed line and the black dot-dashed line correspond to the cases of $l=e, \mu, \tau$, respectively. Here the resonant contributions are not taken into account.

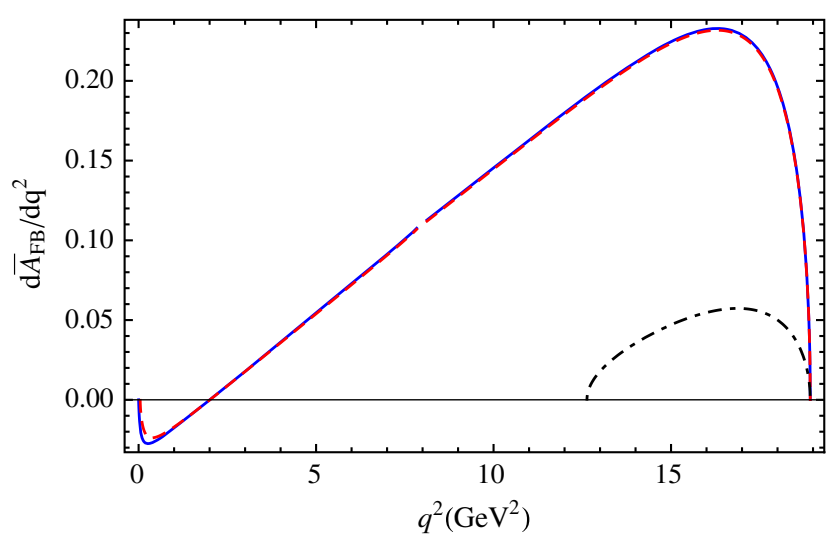

FIG. 4. Same as Fig. 3 but for $d \bar{A}_{F B} / d q^{2}$.

or

$$
\operatorname{Re}\left(C_{9}^{\text {eff }}\left(s_{0}\right)\right)+2 \frac{m_{b} M}{s_{0}} C_{7}^{\text {eff }} \mathcal{R}\left(s_{0}\right)=0 .
$$

Here $\mathcal{R}$ is defined by

$$
\mathcal{R} \equiv \frac{A D-B C}{2 A B}
$$

with

$$
\begin{aligned}
& A=M f_{1}-\left(M+M^{\prime}\right) f_{2}, \\
& B=M g_{1}+\left(M-M^{\prime}\right) g_{2}, \\
& C=M f_{1}^{T}-\left(M+M^{\prime}\right) f_{2}^{T}, \\
& D=M g_{1}^{T}+\left(M-M^{\prime}\right) g_{2}^{T} .
\end{aligned}
$$

The meaning of $\mathcal{R}$ can be seen more clear in $\Lambda_{b} \rightarrow \Lambda$ process with the help of the heavy quark symmetry. In the heavy quark symmetry limit, the matrix elements of all the 
TABLE XIX. Zero-crossing points of $d \bar{A}_{F B} / d q^{2}$ and $\mathcal{R}$ defined in Eqs. (51) and (52) for $b \rightarrow s$ process with $l=e / \mu$.

\begin{tabular}{lcccccccc}
\hline \hline Channels & $s_{0} / \mathrm{GeV}^{2}$ & $\mathcal{R}\left(s_{0}\right)$ & Channels & $s_{0} / \mathrm{GeV}^{2}$ & $\mathcal{R}\left(s_{0}\right)$ & $\mathrm{Channels}^{\prime}$ & $s_{0} / \mathrm{GeV}^{2}$ & $\mathcal{R}\left(s_{0}\right)$ \\
\hline$\Xi_{b b}^{0} \rightarrow \Xi_{b}^{0} l^{+} l^{-}$ & 2.01 & 0.30 & $\Xi_{b c}^{+} \rightarrow \Xi_{c}^{+} l^{+} l^{-}$ & 2.80 & 0.61 & $\Xi_{b c}^{\prime+} \rightarrow \Xi_{c}^{+} l^{+} l^{-}$ & 3.12 & 0.68 \\
$\Xi_{b b}^{-} \rightarrow \Xi_{b}^{-} l^{+} l^{-}$ & 2.01 & 0.30 & $\Xi_{b c}^{0} \rightarrow \Xi_{c}^{0} l^{+} l^{-}$ & 2.80 & 0.61 & $\Xi_{b c}^{\prime 0} \rightarrow \Xi_{c}^{0} l^{+} l^{-}$ & 3.12 & 0.68 \\
$\Xi_{b b}^{0} \rightarrow \Xi_{b}^{\prime \prime} l^{+} l^{-}$ & 2.88 & 0.43 & $\Xi_{b c}^{+} \rightarrow \Xi_{c}^{\prime+} l^{+} l^{-}$ & 3.02 & 0.66 & $\Xi_{b c}^{\prime+} \rightarrow \Xi_{c}^{\prime+} l^{+} l^{-}$ & 2.87 & 0.62 \\
$\Xi_{b b}^{-} \rightarrow \Xi_{b}^{\prime-} l^{+} l^{-}$ & 2.88 & 0.43 & $\Xi_{b c}^{0} \rightarrow \Xi_{c}^{\prime 0} l^{+} l^{-}$ & 3.02 & 0.66 & $\Xi_{b c}^{\prime 0} \rightarrow \Xi_{c}^{\prime 0} l^{+} l^{-}$ & 2.87 & 0.62 \\
$\Omega_{b b}^{-} \rightarrow \Omega_{b}^{-} l^{+} l^{-}$ & 2.88 & 0.42 & $\Omega_{b c}^{0} \rightarrow \Omega_{c}^{0} l^{+} l^{-}$ & 3.00 & 0.65 & $\Omega_{b c}^{\prime 0} \rightarrow \Omega_{c}^{0} l^{+} l^{-}$ & 2.80 \\
\hline \hline
\end{tabular}

TABLE XX. Same as XIX but for $b \rightarrow d$ process.

\begin{tabular}{lcccccccc}
\hline \hline Channels & $s_{0} / \mathrm{GeV}^{2}$ & $\mathcal{R}\left(s_{0}\right)$ & Channels & $s_{0} / \mathrm{GeV}^{2}$ & $\mathcal{R}\left(s_{0}\right)$ & Channels & $s_{0} / \mathrm{GeV}^{2}$ & $\mathcal{R}\left(s_{0}\right)$ \\
\hline$\Xi_{b b}^{0} \rightarrow \Lambda_{b}^{0} l^{+} l^{-}$ & 1.96 & 0.29 & $\Xi_{b c}^{+} \rightarrow \Lambda_{c}^{+} l^{+} l^{-}$ & 2.81 & 0.61 & $\Xi_{b c}^{\prime+} \rightarrow \Lambda_{c}^{+} l^{+} l^{-}$ & 3.09 & 0.67 \\
$\Omega_{b b}^{-} \rightarrow \Xi_{b}^{-} l^{+} l^{-}$ & 2.00 & 0.29 & $\Omega_{b c}^{0} \rightarrow \Xi_{c}^{0} l^{+} l^{-}$ & 2.77 & 0.60 & $\Omega_{b c}^{\prime 0} \rightarrow \Xi_{c}^{0} l^{+} l^{-}$ & 3.11 & 0.67 \\
$\Xi_{b b}^{0} \rightarrow \Sigma_{b}^{0} l^{+} l^{-}$ & 2.88 & 0.43 & $\Xi_{b c}^{+} \rightarrow \Sigma_{c}^{+} l^{+} l^{-}$ & 3.02 & 0.66 & $\Xi_{b c}^{\prime+} \rightarrow \Sigma_{c}^{+} l^{+} l^{-}$ & 2.91 & 0.63 \\
$\Xi_{b b}^{-} \rightarrow \Sigma_{b}^{-} l^{+} l^{-}$ & 2.88 & 0.43 & $\Xi_{b c}^{0} \rightarrow \Sigma_{c}^{0} l^{+} l^{-}$ & 3.02 & 0.66 & $\Xi_{b c}^{\prime 0} \rightarrow \Sigma_{c}^{0} l^{+} l^{-}$ & 2.91 & 0.63 \\
$\Omega_{b b}^{-} \rightarrow \Xi_{b}^{\prime-} l^{+} l^{-}$ & 2.88 & 0.42 & $\Omega_{b c}^{0} \rightarrow \Xi_{c}^{\prime 0} l^{+} l^{-}$ & 3.01 & 0.65 & $\Omega_{b c}^{\prime c} \rightarrow \Xi_{c}^{\prime 0} l^{+} l^{-}$ & 2.84 & 0.61 \\
\hline \hline
\end{tabular}

hadronic currents can be parametrized by only two independent form factors [58]

$$
\left\langle\Lambda\left(p_{\Lambda}\right)|s \Gamma b| \Lambda_{b}\left(p_{\Lambda_{b}}\right)\right\rangle=\bar{u}_{\Lambda}\left[F_{1}\left(q^{2}\right)+\not p F_{2}\left(q^{2}\right)\right] \Gamma u_{\Lambda_{b}},
$$

where $\Gamma$ is the product of Dirac matrices, $v^{\mu} \equiv p_{\Lambda_{b}}^{\mu} / m_{\Lambda_{b}}$ is the four velocity of $\Lambda_{b}$.

Under the heavy quark symmetry,

$$
\begin{aligned}
f_{1}, g_{1}, f_{2}^{T}, g_{2}^{T} & \rightarrow F_{1}, \\
f_{2}, g_{2} & \rightarrow F_{2}, \\
f_{1}^{T}, g_{1}^{T} & \rightarrow 0,
\end{aligned}
$$

and $\mathcal{R}$ is reduced to the following form

$$
\mathcal{R}=\frac{F_{1}^{2}}{F_{1}^{2}-F_{2}^{2}},
$$

where we have also neglected the $m_{\Lambda} / m_{\Lambda_{b}}$ term. If we further take into account the fact that $F_{2} \ll F_{1}$ for $\Lambda_{b} \rightarrow \Lambda$ process [59-61], then

$$
\mathcal{R} \approx 1
$$

The values of $\mathcal{R}$ for FCNC processes of doubly heavy baryons can be found in Tables XIX and XX. It can be seen from these tables that $\mathcal{R}$ roughly ranges from 0.3 to 0.4 for $b b$ sector, while it lies in the interval of $[0.6,0.7]$ for $b c$ sector.

\section{SU(3) analyses}

According to the flavor $\mathrm{SU}(3)$ symmetry, there exist the following relations among these FCNC processes. These relations can be readily derived using the overlapping factors given in Table II. For $b \rightarrow s$ process, we have

$$
\begin{aligned}
\Gamma\left(\Xi_{b b}^{0} \rightarrow \Xi_{b}^{0} l^{+} l^{-}\right) & =\Gamma\left(\Xi_{b b}^{-} \rightarrow \Xi_{b}^{-} l^{+} l^{-}\right) \\
\Gamma\left(\Xi_{b b}^{0} \rightarrow \Xi_{b}^{\prime 0} l^{+} l^{-}\right) & =\Gamma\left(\Xi_{b b}^{-} \rightarrow \Xi_{b}^{\prime-} l^{+} l^{-}\right) \\
& =\frac{1}{2} \Gamma\left(\Omega_{b b}^{-} \rightarrow \Omega_{b}^{-} l^{+} l^{-}\right)
\end{aligned}
$$

for $b b$ sector,

$$
\begin{aligned}
\Gamma\left(\Xi_{b c}^{+} \rightarrow \Xi_{c}^{+} l^{+} l^{-}\right) & =\Gamma\left(\Xi_{b c}^{0} \rightarrow \Xi_{c}^{0} l^{+} l^{-}\right) \\
\Gamma\left(\Xi_{b c}^{+} \rightarrow \Xi_{c}^{\prime+} l^{+} l^{-}\right) & =\Gamma\left(\Xi_{b c}^{0} \rightarrow \Xi_{c}^{\prime 0} l^{+} l^{-}\right) \\
& =\frac{1}{2} \Gamma\left(\Omega_{b c}^{0} \rightarrow \Omega_{c}^{0} l^{+} l^{-}\right)
\end{aligned}
$$

for $b c$ sector and

$$
\begin{aligned}
\Gamma\left(\Xi_{b c}^{\prime+} \rightarrow \Xi_{c}^{+} l^{+} l^{-}\right) & =\Gamma\left(\Xi_{b c}^{\prime 0} \rightarrow \Xi_{c}^{0} l^{+} l^{-}\right) \\
\Gamma\left(\Xi_{b c}^{\prime+} \rightarrow \Xi_{c}^{\prime+} l^{+} l^{-}\right) & =\Gamma\left(\Xi_{b c}^{\prime 0} \rightarrow \Xi_{c}^{\prime 0} l^{+} l^{-}\right) \\
& =\frac{1}{2} \Gamma\left(\Omega_{b c}^{\prime 0} \rightarrow \Omega_{c}^{0} l^{+} l^{-}\right)
\end{aligned}
$$

for $b c^{\prime}$ sector. 
TABLE XXI. Quantitative predictions of SU(3) symmetry breaking for $b \rightarrow s$ process in $b b$ sector.

\begin{tabular}{lccc}
\hline \hline Channels & $\begin{array}{c}\Gamma / \mathrm{GeV} \\
(\mathrm{LFQM})\end{array}$ & $\begin{array}{c}\Gamma / \mathrm{GeV} \\
(\mathrm{SU}(3))\end{array}$ & $\begin{array}{c}|\mathrm{LFQM}-\mathrm{SU}(3)| / \\
\mathrm{SU}(3)\end{array}$ \\
\hline$\Xi_{b b}^{0} \rightarrow \Xi_{b}^{\prime 0} e^{+} e^{-}$ & $5.20 \times 10^{-19}$ & $5.20 \times 10^{-19}$ & $\ldots$ \\
$\Xi_{b b}^{-} \rightarrow \Xi_{b}^{\prime} e^{+} e^{-}$ & $5.20 \times 10^{-19}$ & $5.20 \times 10^{-19}$ & $0 \%$ \\
$\Omega_{b b}^{-} \rightarrow \Omega_{b}^{-} e^{+} e^{-}$ & $1.02 \times 10^{-18}$ & $1.04 \times 10^{-18}$ & $2 \%$ \\
$\Xi_{b b}^{0} \rightarrow \Xi_{b}^{\prime 0} \mu^{+} \mu^{-}$ & $4.47 \times 10^{-19}$ & $4.47 \times 10^{-19}$ & $\ldots$ \\
$\Xi_{b b}^{-} \rightarrow \Xi_{b}^{\prime-} \mu^{+} \mu^{-}$ & $4.47 \times 10^{-19}$ & $4.47 \times 10^{-19}$ & $0 \%$ \\
$\Omega_{b b}^{-} \rightarrow \Omega_{b}^{-} \mu^{+} \mu^{-}$ & $8.85 \times 10^{-19}$ & $8.94 \times 10^{-19}$ & $1 \%$ \\
$\Xi_{b b}^{0} \rightarrow \Xi_{b}^{\prime 0} \tau^{+} \tau^{-}$ & $4.87 \times 10^{-20}$ & $4.87 \times 10^{-20}$ & $\ldots$ \\
$\Xi_{b b}^{-} \rightarrow \Xi_{b}^{\prime-} \tau^{+} \tau^{-}$ & $4.87 \times 10^{-20}$ & $4.87 \times 10^{-20}$ & $0 \%$ \\
$\Omega_{b b}^{-} \rightarrow \Omega_{b}^{-} \tau^{+} \tau^{-}$ & $1.02 \times 10^{-19}$ & $9.74 \times 10^{-20}$ & $5 \%$ \\
\hline \hline
\end{tabular}

TABLE XXII. Quantitative predictions of SU(3) symmetry breaking for $b \rightarrow s$ process in $b c$ sector.

\begin{tabular}{lccc}
\hline \hline Channels & $\begin{array}{c}\Gamma / \mathrm{GeV} \\
(\mathrm{LFQM})\end{array}$ & $\begin{array}{c}\Gamma / \mathrm{GeV} \\
(\mathrm{SU}(3))\end{array}$ & $\begin{array}{c}|\mathrm{LFQM}-\mathrm{SU}(3)| / \\
\mathrm{SU}(3)\end{array}$ \\
\hline$\Xi_{b c}^{+} \rightarrow \Xi_{c}^{\prime+} e^{+} e^{-}$ & $4.54 \times 10^{-19}$ & $4.54 \times 10^{-19}$ & $\ldots$ \\
$\Xi_{b c}^{0} \rightarrow \Xi_{c}^{\prime 0} e^{+} e^{-}$ & $4.53 \times 10^{-19}$ & $4.54 \times 10^{-19}$ & $0 \%$ \\
$\Omega_{b c}^{0} \rightarrow \Omega_{c}^{0} e^{+} e^{-}$ & $7.42 \times 10^{-19}$ & $9.08 \times 10^{-19}$ & $18 \%$ \\
$\Xi_{b c}^{+} \rightarrow \Xi_{c}^{\prime+} \mu^{+} \mu^{-}$ & $3.97 \times 10^{-19}$ & $3.97 \times 10^{-19}$ & $\ldots$ \\
$\Xi_{b c}^{0} \rightarrow \Xi_{c}^{\prime 0} \mu^{+} \mu^{-}$ & $3.95 \times 10^{-19}$ & $3.97 \times 10^{-19}$ & $1 \%$ \\
$\Omega_{b c}^{0} \rightarrow \Omega_{c}^{0} \mu^{+} \mu^{-}$ & $6.41 \times 10^{-19}$ & $7.94 \times 10^{-19}$ & $19 \%$ \\
$\Xi_{b c}^{+} \rightarrow \Xi_{c}^{\prime+} \tau^{+} \tau^{-}$ & $6.50 \times 10^{-20}$ & $6.50 \times 10^{-20}$ & $\ldots$ \\
$\Xi_{b c}^{0} \rightarrow \Xi_{c}^{\prime 0} \tau^{+} \tau^{-}$ & $6.45 \times 10^{-20}$ & $6.50 \times 10^{-20}$ & $1 \%$ \\
$\Omega_{b c}^{0} \rightarrow \Omega_{c}^{0} \tau^{+} \tau^{-}$ & $9.12 \times 10^{-20}$ & $1.30 \times 10^{-19}$ & $30 \%$ \\
\hline \hline
\end{tabular}

For $b \rightarrow d$ process, we have

$$
\begin{aligned}
\Gamma\left(\Xi_{b b}^{0} \rightarrow \Lambda_{b}^{0} l^{+} l^{-}\right) & =\Gamma\left(\Omega_{b b}^{-} \rightarrow \Xi_{b}^{-} l^{+} l^{-}\right), \\
\Gamma\left(\Xi_{b b}^{0} \rightarrow \Sigma_{b}^{0} l^{+} l^{-}\right) & =\frac{1}{2} \Gamma\left(\Xi_{b b}^{-} \rightarrow \Sigma_{b}^{-} l^{+} l^{-}\right) \\
& =\Gamma\left(\Omega_{b b}^{-} \rightarrow \Xi_{b}^{\prime 0} l^{+} l^{-}\right)
\end{aligned}
$$

for $b b$ sector,

$$
\begin{aligned}
\Gamma\left(\Xi_{b c}^{+} \rightarrow \Lambda_{c}^{+} l^{+} l^{-}\right) & =\Gamma\left(\Omega_{b c}^{0} \rightarrow \Xi_{c}^{0} l^{+} l^{-}\right), \\
\Gamma\left(\Xi_{b c}^{+} \rightarrow \Sigma_{c}^{+} l^{+} l^{-}\right) & =\frac{1}{2} \Gamma\left(\Xi_{b c}^{0} \rightarrow \Sigma_{c}^{0} l^{+} l^{-}\right) \\
& =\Gamma\left(\Omega_{b c}^{0} \rightarrow \Xi_{c}^{\prime 0} l^{+} l^{-}\right)
\end{aligned}
$$

for $b c$ sector and
TABLE XXIII. Quantitative predictions of SU(3) symmetry breaking for $b \rightarrow s$ process in $b c^{\prime}$ sector.

\begin{tabular}{lccc}
\hline \hline Channels & $\begin{array}{c}\Gamma / \mathrm{GeV} \\
(\mathrm{LFQM})\end{array}$ & $\begin{array}{c}\Gamma / \mathrm{GeV} \\
(\mathrm{SU}(3))\end{array}$ & $\begin{array}{c}|\mathrm{LFQM}-\mathrm{SU}(3)| / \\
\mathrm{SU}(3)\end{array}$ \\
\hline$\Xi_{b c}^{\prime+} \rightarrow \Xi_{c}^{\prime+} e^{+} e^{-}$ & $1.27 \times 10^{-19}$ & $1.27 \times 10^{-19}$ & $\ldots$ \\
$\Xi_{b c}^{\prime 0} \rightarrow \Xi_{c}^{\prime 0} e^{+} e^{-}$ & $1.26 \times 10^{-19}$ & $1.27 \times 10^{-19}$ & $1 \%$ \\
$\Omega_{b c}^{\prime 0} \rightarrow \Omega_{c}^{0} e^{+} e^{-}$ & $2.11 \times 10^{-19}$ & $2.54 \times 10^{-19}$ & $17 \%$ \\
$\Xi_{b c}^{\prime+} \rightarrow \Xi_{c}^{\prime+} \mu^{+} \mu^{-}$ & $1.21 \times 10^{-19}$ & $1.21 \times 10^{-19}$ & $\ldots$ \\
$\Xi_{b c}^{\prime 0} \rightarrow \Xi_{c}^{\prime 0} \mu^{+} \mu^{-}$ & $1.20 \times 10^{-19}$ & $1.21 \times 10^{-19}$ & $1 \%$ \\
$\Omega_{b c}^{\prime \prime} \rightarrow \Omega_{c}^{0} \mu^{+} \mu^{-}$ & $2.01 \times 10^{-19}$ & $2.42 \times 10^{-19}$ & $17 \%$ \\
$\Xi_{b c}^{\prime+} \rightarrow \Xi_{c}^{\prime+} \tau^{+} \tau^{-}$ & $2.03 \times 10^{-20}$ & $2.03 \times 10^{-20}$ & $\ldots$ \\
$\Xi_{b c}^{\prime 0} \rightarrow \Xi_{c}^{\prime 0} \tau^{+} \tau^{-}$ & $2.01 \times 10^{-20}$ & $2.03 \times 10^{-20}$ & $1 \%$ \\
$\Omega_{b c}^{\prime 0} \rightarrow \Omega_{c}^{0} \tau^{+} \tau^{-}$ & $2.91 \times 10^{-20}$ & $4.06 \times 10^{-20}$ & $28 \%$ \\
\hline \hline
\end{tabular}

$$
\begin{aligned}
\Gamma\left(\Xi_{b c}^{\prime+} \rightarrow \Lambda_{c}^{+} l^{+} l^{-}\right) & =\Gamma\left(\Omega_{b c}^{\prime 0} \rightarrow \Xi_{c}^{0} l^{+} l^{-}\right), \\
\Gamma\left(\Xi_{b c}^{\prime+} \rightarrow \Sigma_{c}^{+} l^{+} l^{-}\right) & =\frac{1}{2} \Gamma\left(\Xi_{b c}^{\prime 0} \rightarrow \Sigma_{c}^{0} l^{+} l^{-}\right) \\
& =\Gamma\left(\Omega_{b c}^{\prime 0} \rightarrow \Xi_{c}^{\prime 0} l^{+} l^{-}\right)
\end{aligned}
$$

for $b c^{\prime}$ sector.

Quantitative analysis for SU(3) symmetry breaking is given in Tables XXI-XXIII for $b \rightarrow s$ process and some comments on $\mathrm{SU}(3)$ symmetry breaking are given as follows.

(i) $\mathrm{SU}(3)$ symmetry breaking is larger for the $Q s$ diquark involved case than that for the $Q u / Q d$ diquark involved case. Here $Q=b, c$.

(ii) $\mathrm{SU}(3)$ symmetry breaking is larger for the $c q$ diquark involved case than that for the $b q$ diquark involved case. Here $q=u, d, s$.

(iii) $\mathrm{SU}(3)$ symmetry breaking is smaller for $l=e / \mu$ cases than that for $l=\tau$ case. This can be attributed to the much smaller phase space for $l=\tau$ case. Smaller phase space is more sensitive to the variation of the masses of baryons in the initial and final states.

\section{E. Uncertainties}

Also taking the process of $\Xi_{b b}^{0} \rightarrow \Xi_{b}^{0}$ as an example, the uncertainties caused by the model parameters and the single pole assumption will be given in this subsection. The error estimates for the form factors can be found in Table XXIV, in which the errors come from $\beta_{i}, \beta_{f}$, and $m_{d i}$, respectively. The error estimates for the decay widths are listed below:

$$
\begin{aligned}
\Gamma\left(\Xi_{b b}^{0} \rightarrow \Xi_{b}^{0} e^{+} e^{-}\right) & =(1.98 \pm 0.49 \pm 1.21 \pm 0.13 \pm 0.26) \times 10^{-19} \mathrm{GeV}, \\
\Gamma\left(\Xi_{b b}^{0} \rightarrow \Xi_{b}^{0} \mu^{+} \mu^{-}\right) & =(1.92 \pm 0.48 \pm 1.18 \pm 0.14 \pm 0.26) \times 10^{-19} \mathrm{GeV}, \\
\Gamma\left(\Xi_{b b}^{0} \rightarrow \Xi_{b}^{0} \tau^{+} \tau^{-}\right) & =(3.72 \pm 0.96 \pm 2.52 \pm 0.51 \pm 1.28) \times 10^{-20} \mathrm{GeV},
\end{aligned}
$$


TABLE XXIV. Error estimates for the form factors, taking $\Xi_{b b}^{0} \rightarrow \Xi_{b}^{0}$ as an example. The first number is the central value, and the following 3 errors come from $\beta_{i}=\beta_{\Xi_{b b}^{0}}, \beta_{f}=\beta_{\Xi_{b}^{0}}$ and $m_{d i}=m_{(b u)}$, respectively. These parameters are all varied by $10 \%$.

\begin{tabular}{lrrr}
\hline \hline$F$ & $F(0)$ & $F$ & $F(0)$ \\
\hline$f_{1, S}^{\Xi_{b b} \rightarrow \Xi_{b}}$ & $0.141 \pm 0.018 \pm 0.036 \pm 0.002$ & $f_{1, A}^{\Xi_{b b} \rightarrow \Xi_{b}}$ & $0.138 \pm 0.018 \pm 0.035 \pm 0.002$ \\
$f_{2, S}^{\Xi_{b b} \rightarrow \Xi_{b}}$ & $-0.189 \pm 0.039 \pm 0.037 \pm 0.014$ & $f_{2, A}^{\Xi_{b b} \rightarrow \Xi_{b}}$ & $0.132 \pm 0.015 \pm 0.027 \pm 0.029$ \\
$f_{3, S}^{\Xi_{b b} \rightarrow \Xi_{b}}$ & $0.016 \pm 0.009 \pm 0.013 \pm 0.019$ & $f_{3, A}^{\Xi_{b b} \rightarrow \Xi_{b}}$ & $-0.068 \pm 0.006 \pm 0.007 \pm 0.022$ \\
$g_{1, S}^{\Xi_{b b} \rightarrow \Xi_{b}}$ & $0.122 \pm 0.020 \pm 0.025 \pm 0.007$ & $g_{1, A}^{\Xi_{b b} \rightarrow \Xi_{b}}$ & $-0.030 \pm 0.004 \pm 0.007 \pm 0.001$ \\
$g_{2, S}^{\Xi_{b b} \rightarrow \Xi_{b}}$ & $0.056 \pm 0.016 \pm 0.045 \pm 0.030$ & $g_{2, A}^{\Xi_{b b} \rightarrow \Xi_{b}}$ & $-0.055 \pm 0.004 \pm 0.017 \pm 0.006$ \\
$g_{3, S}^{\Xi_{b b} \rightarrow \Xi_{b}}$ & $-0.406 \pm 0.088 \pm 0.225 \pm 0.120$ & $g_{3, A}^{\Xi_{b b} \rightarrow \Xi_{b}}$ & $0.261 \pm 0.019 \pm 0.078 \pm 0.022$ \\
$f_{2, S}^{T, \Xi_{b b} \rightarrow \Xi_{b}}$ & $0.108 \pm 0.016 \pm 0.023 \pm 0.020$ & $f_{2, A}^{T, \Xi_{b b} \rightarrow \Xi_{b}}$ & $-0.066 \pm 0.013 \pm 0.013 \pm 0.010$ \\
$f_{3, S}^{T, \Xi_{b b} \rightarrow \Xi_{b}}$ & $0.091 \pm 0.018 \pm 0.018 \pm 0.013$ & $f_{3, A}^{T, \Xi_{b b} \rightarrow \Xi_{b}}$ & $0.134 \pm 0.024 \pm 0.026 \pm 0.011$ \\
$g_{2, S}^{T, \Xi_{b b} \rightarrow \Xi_{b}}$ & $0.128 \pm 0.012 \pm 0.036 \pm 0.002$ & $g_{2, A}^{T, \Xi_{b b} \rightarrow \Xi_{b}}$ & $-0.049 \pm 0.005 \pm 0.012 \pm 0.001$ \\
$g_{3, S}^{T, \Xi_{b b} \rightarrow \Xi_{b}}$ & $0.156 \pm 0.122 \pm 0.020 \pm 0.012$ & $g_{3, A}^{T, \Xi_{b b} \rightarrow \Xi_{b}}$ & $0.032 \pm 0.010 \pm 0.012 \pm 0.002$ \\
\hline \hline
\end{tabular}

where these errors come from $\beta_{i}, \beta_{f}, m_{d i}$, and $m_{\text {pole }}$, respectively. The first three model parameters are all varied by $10 \%$, while $m_{\text {pole }}$, which is responsible for the single pole assumption, is varied by $5 \%$. It can be seen from Table XXIV and Eq. (63) that, the uncertainties caused by these parameters may be sizable.

\section{CONCLUSIONS}

In our previous work, we have investigated the weak decays of doubly heavy baryons for $1 / 2$ to $1 / 2$ case and for $1 / 2$ to $3 / 2$ case. As a continuation, we investigate the FCNC processes in this work. Light-front approach under the diquark picture is once again adopted to extract the form factors. The same method was applied to study the singly heavy baryon decays and reasonable results were obtained [62]. The extracted form factors are then applied to study some observables in these FCNC processes.
We find that most of the branching ratios for $b \rightarrow s$ processes are $10^{-8}-10^{-7}$, while those for $b \rightarrow d$ processes are $10^{-9}-10^{-8}$, which are roughly one order of magnitude smaller than those in mesonic sector. This is because we believe that the lifetime of the doubly heavy baryon is roughly one order of magnitude smaller than that of $B$ meson. SU(3) symmetry and sources of symmetry breaking are discussed. The error estimates are also investigated.

\section{ACKNOWLEDGMENTS}

The authors are grateful to Prof. Wei Wang for valuable discussions and constant encouragements. This work is supported in part by National Natural Science Foundation of China under Grants No. 11575110, No. 11655002, and No. 11735010, Natural Science Foundation of Shanghai under Grant No. 15DZ2272100.
[1] R. Aaij et al. (LHCb Collaboration), Observation of the Doubly Charmed Baryon $\Xi_{c c}^{++}$, Phys. Rev. Lett. 119, 112001 (2017).

[2] H. X. Chen, Q. Mao, W. Chen, X. Liu, and S. L. Zhu, Establishing low-lying doubly charmed baryons, Phys. Rev. D 96, 031501 (2017); Erratum, Phys. Rev. D 96, 119902(E) (2017).

[3] F. S. Yu, H. Y. Jiang, R. H. Li, C. D. Lü, W. Wang, and Z. X. Zhao, Discovery potentials of doubly charmed baryons, Chin. Phys. C 42, 051001 (2018).

[4] W. Wang, F. S. Yu, and Z. X. Zhao, Weak decays of doubly heavy baryons: The $1 / 2 \rightarrow 1 / 2$ case, Eur. Phys. J. C 77, 781 (2017).
[5] Z.X. Zhao, Weak decays of doubly heavy baryons: The $1 / 2 \rightarrow 3 / 2$ case, arXiv:1805.10878.

[6] H. S. Li, L. Meng, Z. W. Liu, and S. L. Zhu, Magnetic moments of the doubly charmed and bottom baryons, Phys. Rev. D 96, 076011 (2017).

[7] L. Meng, N. Li, and S. 1. Zhu, Possible hadronic molecules composed of the doubly charmed baryon and nucleon, arXiv:1707.03598.

[8] W. Wang, Z. P. Xing, and J. Xu, Weak decays of doubly heavy baryons: SU(3) analysis, Eur. Phys. J. C 77, 800 (2017).

[9] M. Karliner and J.L. Rosner, Discovery of DoublyCharmed $\Xi_{c c}$ Baryon Implies a Stable $(b b \bar{u} \bar{d})$ Tetraquark, Phys. Rev. Lett. 119, 202001 (2017). 
[10] T. Gutsche, M. A. Ivanov, J. G. Krner, and V. E. Lyubovitskij, Decay chain information on the newly discovered double charm baryon state $\Xi_{c c}^{++}$, Phys. Rev. D 96, 054013 (2017).

[11] H. S. Li, L. Meng, Z. W. Liu, and S. L. Zhu, Radiative decays of the doubly charmed baryons in chiral perturbation theory, Phys. Lett. B 777, 169 (2018).

[12] Z. H. Guo, Prediction of exotic doubly charmed baryons within chiral effective field theory, Phys. Rev. D 96, 074004 (2017).

[13] Q. F. Lü, K. L. Wang, L. Y. Xiao, and X. H. Zhong, Mass spectra and radiative transitions of doubly heavy baryons in a relativized quark model, Phys. Rev. D 96, 114006 (2017).

[14] L. Y. Xiao, K. L. Wang, Q.f. Lu, X. H. Zhong, and S. L. Zhu, Strong and radiative decays of the doubly charmed baryons, Phys. Rev. D 96, 094005 (2017).

[15] N. Sharma and R. Dhir, Estimates of W-exchange contributions to $\Xi_{c c}$ decays, Phys. Rev. D 96, 113006 (2017).

[16] Y. L. Ma and M. Harada, Chiral partner structure of doubly heavy baryons with heavy quark spin-flavor symmetry, J. Phys. G 45, 075006 (2018).

[17] L. Meng, H.S. Li, Z. W. Liu, and S. L. Zhu, Magnetic moments of the spin- $\frac{3}{2}$ doubly heavy baryons, Eur. Phys. J. C 77, 869 (2017).

[18] R. H. Li, C. D. Lü, W. Wang, F. S. Yu, and Z. T. Zou, Doubly-heavy baryon weak decays: $\Xi_{b c}^{0} \rightarrow p K^{-}$and $\Xi_{c c}^{+} \rightarrow \Sigma_{c}^{++}(2520) K^{-}$, Phys. Lett. B 767, 232 (2017).

[19] C. Y. Wang, C. Meng, Y. Q. Ma, and K. T. Chao, NLO effects for doubly heavy baryon in QCD sum rules, arXiv: 1708.04563.

[20] Y. J. Shi, W. Wang, Y. Xing, and J. Xu, Weak decays of doubly heavy baryons: Multi-body decay channels, Eur. Phys. J. C 78, 56 (2018).

[21] X. H. Hu, Y. L. Shen, W. Wang, and Z. X. Zhao, Weak decays of doubly heavy baryons: "Decay constants", arXiv:1711.10289.

[22] W. Wang and J. Xu, Weak decays of triply heavy baryons, Phys. Rev. D 97, 093007 (2018).

[23] R. Aaij et al. (LHCb Collaboration), First Measurement of the Lifetime of the Doubly Charmed Baryon $\Xi_{c c}^{++}$, Phys. Rev. Lett. 121, 052002 (2018).

[24] R. Aaij et al. (LHCb Collaboration), First observation of the doubly charmed baryon decay $\Xi_{c c}^{++} \rightarrow \Xi_{c}^{+} \pi^{+}$, arXiv:1807 .01919 .

[25] M. T. Traill (LHCb Collaboration), Spectroscopy of doublyheavy baryons at LHCb, Proc. Sci. Hadron2017 (2018) 067.

[26] Z. S. Brown, W. Detmold, S. Meinel, and K. Orginos, Charmed bottom baryon spectroscopy from lattice QCD, Phys. Rev. D 90, 094507 (2014).

[27] W. Jaus, Covariant analysis of the light front quark model, Phys. Rev. D 60, 054026 (1999).

[28] W. Jaus, Semileptonic decays of B and D mesons in the light front formalism, Phys. Rev. D 41, 3394 (1990).

[29] W. Jaus, Relativistic constituent quark model of electroweak properties of light mesons, Phys. Rev. D 44, 2851 (1991).

[30] H. Y. Cheng, C. Y. Cheung, and C. W. Hwang, Mesonic form-factors and the Isgur-Wise function on the light front, Phys. Rev. D 55, 1559 (1997).

[31] H. Y. Cheng, C. K. Chua, and C. W. Hwang, Covariant light front approach for $\mathrm{s}$ wave and $\mathrm{p}$ wave mesons: Its application to decay constants and form-factors, Phys. Rev. D 69, 074025 (2004).

[32] H. Y. Cheng and C. K. Chua, Covariant light front approach for $B \rightarrow K * \gamma, K(1) \gamma, K *(2) \gamma$ decays, Phys. Rev. D 69 , 094007 (2004); Erratum, Phys. Rev. D 81, 059901(E) (2010).

[33] H. W. Ke, X. Q. Li, and Z. T. Wei, Whether new data on $D(s) \rightarrow f(0)(980) e+n u(e)$ can be understood if $\mathrm{f}(0)$ (980) consists of only the conventional q anti-q structure, Phys. Rev. D 80, 074030 (2009).

[34] H. W. Ke, X. Q. Li, and Z. T. Wei, Determining the $\eta-\eta^{\prime}$ mixing by the newly measured $\operatorname{BR}\left(D\left(D_{s}\right) \rightarrow \eta\left(\eta^{\prime}\right)+\right.$ $\bar{l}+\nu_{l}$, Eur. Phys. J. C 69, 133 (2010).

[35] H. Y. Cheng and C. K. Chua, B to V, A, T tensor form factors in the covariant light-front approach: Implications on radiative B decays, Phys. Rev. D 81, 114006 (2010); Erratum, Phys. Rev. D 82, 059904(E) (2010).

[36] C. D. Lu, W. Wang, and Z.T. Wei, Heavy-to-light form factors on the light cone, Phys. Rev. D 76, 014013 (2007).

[37] W. Wang, Y. L. Shen, and C. D. Lu, The study of $B(c)-\rightarrow$ $X(3872) \pi-(K-)$ decays in the covariant light-front approach, Eur. Phys. J. C 51, 841 (2007).

[38] W. Wang, Y. L. Shen, and C. D. Lu, Covariant light-front approach for B(c) transition form factors, Phys. Rev. D 79, 054012 (2009).

[39] W. Wang and Y. L. Shen, $D s \rightarrow K, K *, \phi$ form factors in the covariant light-front approach and exclusive Ds decays, Phys. Rev. D 78, 054002 (2008).

[40] X. X. Wang, W. Wang, and C. D. Lu, B(c) to P-wave charmonia transitions in covariant light-front approach, Phys. Rev. D 79, 114018 (2009).

[41] C. H. Chen, Y. L. Shen, and W. Wang, $|V(u b)|$ and $B \rightarrow \eta\left(^{\prime}\right)$ form factors in covariant light front approach, Phys. Lett. B 686, 118 (2010).

[42] G. Li, F. 1. Shao, and W. Wang, $B_{s} \rightarrow D_{s}$ (3040) form factors and $B_{s}$ decays into $D_{s}(3040)$, Phys. Rev. D 82, 094031 (2010).

[43] R. C. Verma, Decay constants and form factors of s-wave and p-wave mesons in the covariant light-front quark model, J. Phys. G 39, 025005 (2012).

[44] Y. J. Shi, W. Wang, and Z. X. Zhao, $B_{c} \rightarrow B_{s J}$ form factors and $B_{c}$ decays into $B_{S J}$ in covariant light-front approach, Eur. Phys. J. C 76, 555 (2016).

[45] H. W. Ke, X.Q. Li, and Z.T. Wei, Diquarks and $\Lambda(b) \rightarrow \Lambda(c)$ weak decays, Phys. Rev. D 77, 014020 (2008).

[46] Z. T. Wei, H. W. Ke, and X. Q. Li, Evaluating decay rates and asymmetries of $\Lambda(b)$ into light baryons in LFQM, Phys. Rev. D 80, 094016 (2009).

[47] H. W. Ke, X. H. Yuan, X. Q. Li, Z. T. Wei, and Y. X. Zhang, $\Sigma_{b} \rightarrow \Sigma_{c}$ and $\Omega_{b} \rightarrow \Omega_{c}$ weak decays in the light-front quark model, Phys. Rev. D 86, 114005 (2012).

[48] J. Zhu, Z. T. Wei, and H. W. Ke, The semi-leptonic and nonleptonic weak decays of $\Lambda_{b}^{0}$, arXiv:1803.01297.

[49] H. W. Ke and X. Q. Li, $\Sigma_{b} \rightarrow \Sigma_{c}^{*}$ weak decays in the lightfront quark model, arXiv:1711.02518.

[50] G. Buchalla, A. J. Buras, and M. E. Lautenbacher, Weak decays beyond leading logarithms, Rev. Mod. Phys. 68, 1125 (1996). 
[51] R. H. Li, C. D. Lu, and W. Wang, Branching ratios, forwardbackward asymmetry and angular distributions of $B \rightarrow$ $K(1) l+l$ - decays, Phys. Rev. D 79, 094024 (2009).

[52] C. D. Lu and W. Wang, Analysis of $B \rightarrow K_{J}^{*}(\rightarrow K \pi) \mu^{+} \mu^{-}$in the higher kaon resonance region, Phys. Rev. D 85, 034014 (2012).

[53] A. K. Giri and R. Mohanta, Study of FCNC mediated Z boson effect in the semileptonic rare baryonic decays $\Lambda(b) \rightarrow \Lambda l+l-$, Eur. Phys. J. C 45, 151 (2006).

[54] A.J. Buras and M. Munz, Effective Hamiltonian for $B \rightarrow X(s) e+e-$ beyond leading logarithms in the NDR and HV schemes, Phys. Rev. D 52, 186 (1995).

[55] M. Karliner and J. L. Rosner, Baryons with two heavy quarks: Masses, production, decays, and detection, Phys. Rev. D 90, 094007 (2014).

[56] V. V. Kiselev and A. K. Likhoded, Usp. Fiz. Nauk 172, 497 (2002) [Baryons with two heavy quarks, Phys. Usp. 45, 455 (2002)].
[57] C. Patrignani et al. (Particle Data Group), Review of particle physics, Chin. Phys. C 40, 100001 (2016).

[58] A. V. Manohar and M. B. Wise, Heavy quark physics, Cambridge Monogr. Part. Phys., Nucl. Phys., Cosmol. 10, 1 (2000).

[59] C. S. Huang and H. G. Yan, Exclusive rare decays of heavy baryons to light baryons: $\Lambda(b) \rightarrow \Lambda \gamma$ and $\Lambda(b) \rightarrow \Lambda l+l-$, Phys. Rev. D 59, 114022 (1999); Erratum, Phys. Rev. D 61, 039901(E) (2000).

[60] C. H. Chen and C. Q. Geng, Lepton asymmetries in heavy baryon decays of $\Lambda(b) \rightarrow$ Nepton + lepton-, Phys. Lett. B 516, 327 (2001).

[61] C. H. Chen and C. Q. Geng, Rare $\Lambda(b) \rightarrow$ Alepton + lepton- decays with polarized lambda, Phys. Rev. D 63, 114024 (2001).

[62] Z.X. Zhao, Weak decays of heavy baryons in light-front approach, arXiv:1803.02292. 